\title{
Industry Portfolio Allocation with Asymmetric Correlations ${ }^{\ddagger}$
}

\author{
Myeong Hyeon Kim*, Seyoung Park ${ }^{\dagger}$, and Jong Mun Yoon ${ }^{+}$ \\ *Seoul National University of Science and Technology, E-mail: mhkim@seoultech.ac.kr \\ ${ }^{\dagger}$ Nottingham University Business School, University of Nottingham, E-mail: \\ seyoung.park@nottingham.ac.uk \\ ${ }^{+}$Corresponding Author: The Credit Finance Research Institute, The Credit Finance Association of \\ Korea, Hanway Building, 70 Da-Dong, Jung-gu, Seoul 04521, Korea, E-mail: idisyun@gmail.com \\ $\ddagger$ The authors are grateful for the helpful discussions with Andrew Vivian and participants at the \\ Workshop on Recent Developments in Financial Data Science and Econometrics. The research of the \\ second author was supported by the Ministry of Education of the Republic of Korea and the National \\ Research Foundation of Korea (NRF-2019S1A5A2A03054249).
}

\begin{abstract}
We develop a new framework of optimal consumption and portfolio choice at industry portfolio level under dynamic and asymmetric correlations between industry and market portfolios. We derive in closed-form the optimal consumption and investment strategies under regime-dependent correlations environment. Overall, we find that ignoring timevarying and asymmetric correlations between portfolios can be costly to investors when applied to a construction of the optimal portfolio. Finally, we empirically test the performance of the model-based investment strategy.

Keywords: Industry Portfolio, Regime Switching, Dynamic and Asymmetric Correlation JEL Classification: G11, G12, C61
\end{abstract}

\section{Introduction}

What determines a satisfactory investment portfolio? Markowitz (1959) and Merton $(1969,1971)$ provide a benchmark against which investment decisions can be rationalized: the risk (or volatility) and return must be balanced optimally. In this framework, an opti- 
mal investment decision is to achieve the best risk-return combination across all attainable combinations of risk and return (or in the entire investment opportunity set) offered by portfolios.

Standard investment literature (e.g., Markowitz, 1959; Merton, 1969, 1971) assumes that the investment opportunity is constant, and therefore faces a limitation by neglecting one major dimension of financial risk: time-varying and asymmetric correlations between portfolios. In our study, we overcome this limitation and solve the optimal consumption and investment model in a regime switching market with regime-dependent correlations between portfolios. We hope this paper will lend itself to the study of investment strategies for investors, industry, and academic professions as well.

Since the seminal work of Hamilton (1989), many studies have widely adopted his regime switching framework in economic modeling. As Cochrane (2017) arguably states that

Asset prices and returns are correlated with business cycles. Stocks rise in good times, and fall in bad times. Real and nominal interest rates rise and fall with the business cycle. Stock returns and bond yield also help to forecast macroeconomic events such as GDP growth and inflation.

As to the regime switching model applications to the investment strategies, Ang and Bekaert (2002) thoroughly investigate international asset allocation with regime switching. Jang et al. (2007) use the regime switching model showing that the variability of an investment opportunity significantly changes the optimal consumption and investment 
decisions in the presence of transaction costs. Liu and Loewenstein (2013) take market crashes into account the asset allocation framework by extending Jang et al. (2007)'s regime switching model. Dai et al. (2016) investigate the effects of market closure on optimal investment when an investment opportunity set fluctuates across regimes. More recently, Dai et al. (2018) study the effects of capital gains tax on portfolio selection using the regime switching framework.

Our work also sits squarely within such a regime switching model application with empirical evidence supporting our modeling of regime-switching correlations across portfolios. While existing symmetric models do not distinguish two different market situations and calculate correlations between portfolios, our asymmetric model does consider two different market environments as the bull market and the bear market, and calculates time-varying and asymmetric correlations. Indeed, the conditional correlations between industry and market portfolio are time varying and asymmetric, relying on the current state of the regime: the bull regime or the bear regime (Figure C.1).1 More specifically, we support the existence of substantial heterogeneity across magnitudes of conditional correlations. For example, correlations between S\&P 500 and health industry show a dramatic plunge at times of economic recessions, while correlations between S\&P 500 and hi-tech industry show a modest decrease. We also find that the presence of the asymmetric dynamic conditional correlation (ADCC) expands the efficient frontier, and thus the investment

\footnotetext{
${ }^{1}$ In this empirical analysis, we have applied the asymmetric dynamic conditional correlation (ADCC) to estimate the conditional correlations (Cappiello et al., 2006).
} 
opportunities under a dynamic mean-variance framework (Figure C.2) $\mathrm{L}^{2}$

\section{[Insert Figure C.1 here.]}

\section{[Insert Figure C.2 here.]}

In this paper, we develop a new framework of optimal consumption and portfolio choice at industry portfolio level under dynamic and asymmetric correlations between industry and market portfolios. We derive in closed-form the optimal consumption and investment strategies under regime-dependent correlations environment. We carry out an in-depth quantitative analysis to illustrate various properties of the optimal strategies. We find that time variations in correlation in the investment opportunity set, depending upon the current status of the regime, can play a role in explaining the asset pricing implications further. Intuitively, the risk associated with random fluctuations in correlation cannot be fully diversified and hence, investors should require a premium to compensate for their exposure to dynamic and asymmetric correlation. Such a risk compensation, thus, should be reflected in the optimal investment portfolio, adjusting its amount substantially in response to variations in correlation across the bull and bear regimes.

We empirically evaluate whether our model-based investment strategy can generate meaningful performance. We first provide empirical evidence supporting the use of regimedependent correlation for managing portfolios. Similar to Gomes et al. (2009), we consider two industry portfolios: durable sector A and non-durable sector B. We then examine the

\footnotetext{
${ }^{2}$ The low risk - low return area displays insignificant differences, whereas the high risk - high return area presents a clear expansion heading to the northwest. Further, Figure C.2 supports the existence of substantial heterogeneity on risk-return profiles across different industries.
} 
empirical performance of our proposed investment strategy, compared to other heuristic strategies such as $1 / n$, maximum diversification, inverse volatility, equal risk parity, and two tail-risk parity strategies.

In the empirical application, we find that correlations between consumer durables and non-durables are inclined to rise at times of economic recessions including Great Depression (1929-1939), Oil Shock (1973-1974), and Global Financial Crisis (2007-2009). We also find that our proposed portfolio incorporating regime-dependent dynamic correlations outperforms, especially during the Global Financial Crisis period, other heuristic portfolios in the aspect of cumulative return, standard deviation, Sharpe ratio, and maximum drawdown. Our analysis therefore suggests that consideration of dynamic and asymmetric correlations between industry and market portfolios is an important factor in the attainment of successful investment return in the crisis period. Loosely speaking, the greater the change in the investment opportunity set after regime switching from the bull (bear) regime to the bear (bull) regime just as economic recessions, the greater the benefit of considering timevarying correlation dynamics. This inversely implies that misestimating or overlooking such a regime-dependent correlation can be costly to investors. For instance, if an investor underestimates the correlation and adopts the corresponding heuristic investment strategies under the wrong estimation, the expected wealth loss from these trading strategies would be very high.

Closely related works include the literature on sector-level investment without correlation fluctuations (e.g., Gomes et al., 2009; Phylaktis and Xia 2009; Kalotychou et al., 2014; Chava et al., 2018), and the literature on portfolio selection with correlation fluc- 
tuations but without sector-level investment (e.g. Buraschi et al., 2010). Existing works, however, do not consider sector-level investment and correlation fluctuations jointly. It is thus, unclear what a model of optimal investment with such a joint consideration would deliver. We view our analytically tractable model as a complement for our better understanding of mainly numerically solved existing models with either sector-level investment or time-varying and asymmetric correlations between portfolios.

The paper is organized as follows. In Section 2, we develop a model of optimal consumption and portfolio choice under a regime switching environment with regime-dependent correlations between portfolios. In Section 3, we derive analytically tractable results for the optimal consumption and investment strategies. In Section 4, we carry out an in-depth quantitative analysis to investigate various properties of the optimal strategies further. In

Section 5, we empirically test the performance of the model-based investment strategy. In Section 6, we conclude the paper.

\section{The Model}

Utility Function. As proposed by Duffie and Epstein (1992), an investor has the following continuous-time formulation of non-expected utility:

$$
V_{t}=E_{t}\left[\int_{t}^{\infty} f\left(c_{s}, V_{s}\right) d s\right],
$$


where $E_{t}$ is the expectation taken at time $t$ and $f(c, V)$ is the normalized aggregator for consumption $c$ and utility $V$. The aggregator $f(c, V)$ follows

$$
f(c, V)=\frac{\rho^{*}}{1-\psi^{-1}} \frac{c^{1-\psi^{-1}}-((1-\gamma) V)^{\theta}}{((1-\gamma) V)^{\theta-1}}
$$

and

$$
\theta=\frac{1-\psi^{-1}}{1-\gamma}
$$

Here, $\psi>0$ is the elasticity of intertemporal substitution (EIS), $\gamma>0$ is the coefficient of relative risk aversion (CRRA), and $\rho^{*}>0$ is the subjective discount rate. When $\gamma=\psi^{-1}$, implying $\theta=1$, the recursive utility $f(c, V)$ reduces to the widely used time-additive separable CRRA utility. In this case, $f(c, V)=U(c)-\rho^{*} V$ with $U(c)=\rho^{*} c^{1-\gamma} /(1-\gamma)$ and thus, it is additively separable in $c$ and $V$. For $\theta \neq 1$, the general specification of the recursive utility $f(c, V)$ is non-separable in $c$ and $V$.

A Regime Switching Model. To examine the impact of macroeconomic conditions on optimal investment in the simplest possible environment, we assume that there are two regimes: "Bull" (regime 0) and "bear" (regime 1). The fundamental parameters in the financial market are regime dependent. We let $i \in\{0,1\}$ denote the current state of the regime, which is assumed to be governed by a two-state Markov chain with generators as 
the following:

$$
\left(\begin{array}{cc}
\lambda_{0} & -\lambda_{0} \\
-\lambda_{1} & \lambda_{1}
\end{array}\right)
$$

Similar to Jang et al. (2007), we assume that an investor can observe the regime changing. According to the assumed two-state Markov chain, regime $i$ switches into regime $j$ at the first jump time of a Poisson jump process with intensity $\lambda_{i}>0$, for $i, j \in\{0,1\}$. Within the present model, the time $T_{i}$ to leave regime $i$ follows an exponential distribution with intensity $\lambda_{i}$ :

$$
\text { probability of }\left\{T_{i}>t\right\}=e^{-\lambda_{i} t}, \quad i \in\{0,1\}
$$

which implies that there is some probability of $\lambda_{i} d t$ that regime $i$ switches into regime $j$ over an infinitesimal time interval $d t$. Note that the expected duration of regime $i$ is $1 / \lambda_{i}$ and the average fraction of time spent in regime $i$ is $\lambda_{j} /\left(\lambda_{i}+\lambda_{j}\right)$.

Financial Market. An investor can trade the following assets in the financial market. In regime $i(i \in\{0,1\})$, the investor can invest in a bond (or a risk-free asset) growing at a continuously compounded, constant rate $r_{i}>0$. She can also trade three risky assets: Public market portfolio $M$, Industry A stock, and Industry B stock. In regime $i$, the value of public market portfolio, $S_{t}^{M}$, follows the widely adopted geometric Brownian motion $(\mathrm{GBM})$ :

$$
d S_{t}^{M}=\mu_{i}^{M} S_{t}^{M} d t+\sigma_{i}^{M} S_{t}^{M} d W_{t}^{M}
$$

where $\mu_{i}^{M}\left(\mu_{i}^{M}>r_{i}\right)$ and $\sigma_{i}^{M}\left(\sigma_{i}^{M}>0\right)$ are the regime-dependent drift and volatility 
parameters, respectively, and $W_{t}^{M}$ is a standard Brownian motion defined on a suitable probability space. The price process of Industry A stock, $S_{t}^{A}$, follows the GBM:

$$
d S_{t}^{A}=\mu_{i}^{A} S_{t}^{A} d t+\sigma_{i}^{A} S_{t}^{A} d W_{t}^{A}
$$

where $\mu_{i}^{A}\left(\mu_{i}^{A}>r_{i}\right)$ is the expected rate of Industry A stock return, $\sigma_{i}^{A}\left(\sigma_{i}^{A}>0\right)$ is the volatility of the return on Industry A stock, and $W_{t}^{A}$ is a standard Brownian motion with a correlation $\tilde{\rho}_{i}^{A}\left(\left|\tilde{\rho}_{i}^{A}\right|<1\right)$ with $W_{t}^{M}$. Similarly, the price process of Industry B stock, $S_{t}^{B}$, evolves by the following GBM:

$$
d S_{t}^{B}=\mu_{i}^{B} S_{t}^{B} d t+\sigma_{i}^{B} S_{t}^{B} d W_{t}^{B}
$$

where $\mu_{i}^{B}\left(\mu_{i}^{B}>r_{i}\right)$ is the expected rate of Industry B stock return, $\sigma_{i}^{B}\left(\sigma_{i}^{B}>0\right)$ is the volatility of the return on Industry B stock, and $W_{t}^{B}$ is a standard Brownian motion with a correlation $\tilde{\rho}_{i}^{B}\left(\left|\tilde{\rho}_{i}^{B}\right|<1\right)$ with $W_{t}^{M}$, and a correlation $\rho_{i}\left(\left|\rho_{i}\right|<1\right)$ with $W_{t}^{A}$. The Brownian motions $W_{t}^{j}(j \in\{M, A, B\})$ and the Poisson process representing the regime switching risk are assumed to be independent.

In our regime switching model, the investment opportunity is comprised of risk-free interest rate $r_{i}(i \in\{0,1\})$, expected rates $\mu_{i}^{k}(k \in\{M, A, B\})$ of stock returns, volatilities $\sigma_{i}^{k}$ of the returns on stocks, correlation $\tilde{\rho}_{i}^{k}$ between market portfolio and industry portfolio, and correlation $\rho_{i}$ between Industry A and Industry B stocks, which are all regime dependent. 
Systematic Risk. Given the correlations $\left|\tilde{\rho}_{i}^{k}\right|<1(k \in\{A, B\})$ in regime $i(i \in\{0,1\})$ between market portfolio and industry stocks, the risks associated with industry stocks are not fully diversified by only dynamically trading market portfolio. In regime $i(i \in\{0,1\})$, the beta of Industry $k$ stock $(k \in\{A, B\})$ relative to market portfolio can be defined as

$$
\beta_{i}^{k}=\frac{\tilde{\rho}_{i}^{k} \sigma_{i}^{k}}{\sigma_{i}^{M}}
$$

Next, we define the systematic risk of industry stocks in terms of industry beta. The total volatility (or risk) of Industry $k(k \in\{A, B\})$ stock is $\sigma_{i}^{k}$. The part of this volatility (spanned by the market portfolio) is $\tilde{\rho}_{i}^{k} \sigma_{i}^{k}$. The remaining volatility is denoted by $\epsilon_{i}$, which is given by:

$$
\epsilon_{i}=\sqrt{\left(\sigma_{i}^{k}\right)^{2}-\left(\tilde{\rho}_{i}^{k}\right)^{2}\left(\sigma_{i}^{k}\right)^{2}}=\sqrt{\left(\sigma_{i}^{k}\right)^{2}-\left(\beta_{i}^{k}\right)^{2}\left(\sigma_{i}^{M}\right)^{2}} .
$$

The undiversified volatility presents extra risk in an investor's overall portfolio. Thus, the investor requires different risk premia for bearing diversified and undiversified risks.

In the context of risk-return trade-off, an investor is able to earn excess risk-adjusted returns, known as called alphas, by investing in industry stocks. More specifically, the alphas are defined as follows: for Industry $k \in\{A, B\}$,

$$
\alpha_{i}^{k}=\mu_{i}^{k}-r_{i}-\beta_{i}^{k}\left(\mu_{i}^{M}-r_{i}\right) .
$$

Intuitively, the alphas are the capital asset pricing model (CAPM)-model-based risk- 
adjusted excess returns of the portfolio that consists of Industry A and Industry B stocks.

An Optimal Consumption and Portfolio Choice Problem. An investor's optimal consumption and portfolio choice problem is to maximize her recursive utility by controlling per-period consumption $c$ and stock holdings $\pi^{M}, \pi^{A}$, and $\pi^{B}$. This results in the following stochastic optimization problem with a nonnegative wealth constraint: in regime $i$ ( $i \in$ $\{0,1\})$

$$
V^{i}(x) \equiv \max _{\left(c, \pi^{M}, \pi^{A}, \pi^{B}\right)} E_{t}\left[\int_{t}^{\infty}\left\{\frac{\rho^{*}}{1-\psi^{-1}} \frac{c_{s}^{1-\psi^{-1}}-\left((1-\gamma) V_{s}^{i}\right)^{\theta}}{\left((1-\gamma) V_{s}^{i}\right)^{\theta-1}}\right\} d s\right]
$$

subject to the following dynamic wealth constraints: with $X_{0}=x \geq 0$,

$$
\begin{gathered}
d X_{t}=\left(r_{i} X_{t}-c_{t}\right) d t+\pi_{t}^{M} \sigma_{i}^{M}\left(d W_{t}^{M}+\kappa_{i}^{M} d t\right)+\pi_{t}^{A} \sigma_{i}^{A}\left(d W_{t}^{A}+\kappa_{i}^{A} d t\right)+\pi_{t}^{B} \sigma_{i}^{B}\left(d W_{t}^{B}+\kappa_{i}^{B} d t\right), \\
X_{t}>0, \quad \forall t \geq 0 \text { 毒 }
\end{gathered}
$$

where $\pi^{M}$ represents the dollar amount invested in the market portfolio $M$, and $\pi^{A}$ and $\pi^{B}$ are the dollar amount invested in the Industry $A$ and Industry $B$ stocks, respectively. Moreover, $\kappa_{i}^{k}(k \in\{M, A, B\})$ are the Sharpe ratios, $\left(\mu_{i}^{k}-r_{i}\right) / \sigma_{i}^{k}$, in regime $i$.

At time $t$, an investor consumes at the rate equal to $c_{t}$ and receives interest at the rate $r_{i}$ proportional to her wealth by investing in a risk-free bond, resulting in wealth

\footnotetext{
${ }^{3}$ This shows that the investor cannot consume and invest in the financial market any more as her wealth approaches zero. We exclude such a trivial case and thus, only consider the cases in which wealth is above zero so that the investor can consume and invest. We call the consumption and investment strategies satisfying this wealth constraint admissible strategies.
} 
accumulation at the rate of $\left(r_{i} X_{t}-c_{t}\right)$. As the investor is exposed to systematic risk stemming from investments in market portfolio and industry stocks, i.e., when she faces random fluctuations of her wealth given by $\pi_{t}^{k} \sigma_{i}^{k} d W_{t}^{k}$ for $k \in\{M, A, B\}$, the risk taking compensation is $\pi_{t}^{k} \sigma_{i}^{k} \kappa_{i}^{k} d t=\pi_{t}^{k}\left(\mu_{i}^{k}-r_{i}\right) d t$ for $k \in\{M, A, B\}$.

\section{Optimal Strategies}

We derive in closed-form optimal consumption and investment strategies under a regime switching environment, where dynamic and asymmetric correlations between industry and market portfolios are incorporated.

Theorem 3.1. In regime $i(i \in\{0,1\})$, optimal consumption $c_{t}^{*}$ and optimal stock holdings $\left(\pi_{t}^{M}\right)^{*},\left(\pi_{t}^{A}\right)^{*}$, and $\left(\pi_{t}^{B}\right)^{*}$ are derived in closed-form: for any $x>0$,

$$
\begin{aligned}
& c_{t}^{*}=\left(\rho^{*}\right)^{\psi} K_{i}^{-\theta \psi} x, \\
& \left(\pi_{t}^{M}\right)^{*}=\left[\left\{1-\left(\rho_{i}\right)^{2}\right\} \frac{\kappa_{i}^{M}}{\gamma \sigma_{i}^{M}} x+\left(\tilde{\rho}_{i}^{A} \rho_{i} \frac{\sigma_{i}^{B}}{\sigma_{i}^{M}}-\tilde{\rho}_{i}^{B} \frac{\sigma_{i}^{B}}{\sigma_{i}^{M}}\right) \frac{\kappa_{i}^{B}}{\gamma \sigma_{i}^{B}} x\right. \\
& \left.+\left(\tilde{\rho}_{i}^{B} \rho_{i} \frac{\sigma_{i}^{A}}{\sigma_{i}^{M}}-\tilde{\rho}_{i}^{A} \frac{\sigma_{i}^{A}}{\sigma_{i}^{M}}\right) \frac{\kappa_{i}^{A}}{\gamma \sigma_{i}^{A}} x\right] /\left\{1-\left(\rho_{i}\right)^{2}-\left(\tilde{\rho}_{i}^{A}\right)^{2}-\left(\tilde{\rho}_{i}^{B}\right)^{2}+2 \rho_{i} \tilde{\rho}_{i}^{A} \tilde{\rho}_{i}^{B}\right\}, \\
& \left(\pi_{t}^{A}\right)^{*}=\left[\left\{1-\left(\tilde{\rho}_{i}^{B}\right)^{2}\right\} \frac{\kappa_{i}^{A}}{\gamma \sigma_{i}^{A}} x+\left(\tilde{\rho}_{i}^{A} \tilde{\rho}_{i}^{B} \frac{\sigma_{i}^{B}}{\sigma_{i}^{A}}-\rho_{i} \frac{\sigma_{i}^{B}}{\sigma_{i}^{A}}\right) \frac{\kappa_{i}^{B}}{\gamma \sigma_{i}^{B}} x\right. \\
& \left.+\left(\tilde{\rho}_{i}^{B} \rho_{i} \frac{\sigma_{i}^{M}}{\sigma_{i}^{A}}-\tilde{\rho}_{i}^{A} \frac{\sigma_{i}^{M}}{\sigma_{i}^{A}}\right) \frac{\kappa_{i}^{M}}{\gamma \sigma_{i}^{M}} x\right] /\left\{1-\left(\rho_{i}\right)^{2}-\left(\tilde{\rho}_{i}^{A}\right)^{2}-\left(\tilde{\rho}_{i}^{B}\right)^{2}+2 \rho_{i} \tilde{\rho}_{i}^{A} \tilde{\rho}_{i}^{B}\right\}, \\
& \left(\pi_{t}^{B}\right)^{*}=\left[\left\{1-\left(\tilde{\rho}_{i}^{A}\right)^{2}\right\} \frac{\kappa_{i}^{B}}{\gamma \sigma_{i}^{B}} x+\left(\tilde{\rho}_{i}^{A} \tilde{\rho}_{i}^{B} \frac{\sigma_{i}^{A}}{\sigma_{i}^{B}}-\rho_{i} \frac{\sigma_{i}^{A}}{\sigma_{i}^{B}}\right) \frac{\kappa_{i}^{A}}{\gamma \sigma_{i}^{A}} x\right. \\
& \left.+\left(\tilde{\rho}_{i}^{A} \rho_{i} \frac{\sigma_{i}^{M}}{\sigma_{i}^{B}}-\tilde{\rho}_{i}^{B} \frac{\sigma_{i}^{M}}{\sigma_{i}^{B}}\right) \frac{\kappa_{i}^{M}}{\gamma \sigma_{i}^{M}} x\right] /\left\{1-\left(\rho_{i}\right)^{2}-\left(\tilde{\rho}_{i}^{A}\right)^{2}-\left(\tilde{\rho}_{i}^{B}\right)^{2}+2 \rho_{i} \tilde{\rho}_{i}^{A} \tilde{\rho}_{i}^{B}\right\},
\end{aligned}
$$


where $K_{i}$ is a solution to the following system of equations for $i, j \in\{0,1\}$ :

$$
\begin{aligned}
0= & \frac{\psi^{-1}}{1-\psi^{-1}}\left(\rho^{*}\right)^{\psi} K_{i}^{-\theta \psi}-\frac{\rho^{*}}{1-\psi^{-1}}+r_{i}-\frac{1}{2} \gamma\left(\sigma_{i}^{M}\right)^{2}\left(\text { cons }^{M}\right)^{2}+\sigma_{i}^{M} \kappa_{i}^{M} \operatorname{cons}^{M} \\
& -\frac{1}{2} \gamma\left(\sigma_{i}^{A}\right)^{2}\left(\text { cons }^{A}\right)^{2}+\sigma_{i}^{A} \kappa_{i}^{A} \operatorname{cons}^{A}-\frac{1}{2} \gamma\left(\sigma_{i}^{B}\right)^{2}\left(\text { cons }^{B}\right)^{2}+\sigma_{i}^{B} \kappa_{i}^{B} \operatorname{cons}^{B}-\gamma \tilde{\rho}^{A} \sigma_{i}^{M} \sigma_{i}^{A} \operatorname{cons}^{M} \operatorname{cons}^{A} \\
& -\gamma \tilde{\rho}_{i}^{B} \sigma_{i}^{M} \sigma_{i}^{B} \text { cons }^{M} \text { cons }^{B}-\gamma \rho_{i} \sigma_{i}^{A} \sigma_{i}^{B} \operatorname{cons}^{A} \operatorname{cons}^{B}+\lambda_{i}\left(\frac{K_{j}}{K_{i}}-1\right) \frac{1}{1-\gamma}
\end{aligned}
$$

Here, the terms of $\mathrm{cons}^{M}, \mathrm{con}^{A}$, and $\mathrm{con}^{B}$ are constants defined in Appendix A.

Proof. Refer to Appendix A. Q.E.D.

The optimal strategies given in (2) show that consumption and stock holdings have a linear relation with an investor's initial wealth. The investor formulates a non-myopic optimal consumption plan in the sense that future regime changes affect the consumption amount through the regime-dependent constant $K_{i}$ for $i \in\{0,1\}$. Specifically, the consumption strategy is affected by not only the regime intensity $\lambda_{i}$, but also key parameters involving the coefficient $\gamma$ of CRRA and the EIS $\psi$.

Next, we determine some interesting implications of an investor's optimal investment strategies. To have a benchmark, we consider the simplest possible situation in which the market portfolio, Industry A and Industry B stocks are all independent i.e., $\tilde{\rho}_{i}^{k}=\rho_{i}=0$. Then, in regime $i(i \in\{0,1\})$, the optimal stock holdings reduce to the following:

$$
\left(\pi_{t}^{M}\right)^{*}=\frac{\kappa_{i}^{M}}{\gamma \sigma_{i}^{M}} x, \quad\left(\pi_{t}^{A}\right)^{*}=\frac{\kappa_{i}^{A}}{\gamma \sigma_{i}^{A}} x, \quad \text { and }\left(\pi_{t}^{B}\right)^{*}=\frac{\kappa_{i}^{B}}{\gamma \sigma_{i}^{B}} x
$$

These optimal investment strategies follow the traditional investment rule given by Merton 
(1969, 1971): an investor tends to invest more in stocks as the Sharpe ratio $\kappa_{i}^{k}(k \in$ $\{M, A, B\})$ increases or the coefficient $\gamma$ of CRRA decreases.

When we consider only the systematic risk of Industry A and Industry B stocks i.e., $\left|\tilde{\rho}_{i}^{k}\right|<1(k \in\{A, B\}, i \in\{0,1\}), \tilde{\rho}_{i}^{k} \neq 0$, and $\rho_{i}=0$, the market portfolio and Industry A and Industry B stocks are not perfectly correlated. The risks associated with industry stocks are not fully diversified by dynamically trading only the bond and market portfolio. Such undiversified risks have a significant influence on an investor's overall optimal portfolio strategy, inducing extra demand for hedging. More precisely, the optimal investment strategy reduces to the following:

$$
\begin{gathered}
\left(\pi_{t}^{M}\right)^{*}=\left[\frac{\kappa_{i}^{M}}{\gamma \sigma_{i}^{M}} x-\tilde{\rho}_{i}^{B} \frac{\kappa_{i}^{B}}{\gamma \sigma_{i}^{M}} x-\tilde{\rho}_{i}^{A} \frac{\kappa_{i}^{A}}{\gamma \sigma_{i}^{M}} x\right] /\left\{1-\left(\tilde{\rho}_{i}^{A}\right)^{2}-\left(\tilde{\rho}_{i}^{B}\right)^{2}\right\}, \\
\left(\pi_{t}^{A}\right)^{*}=\left[\left\{1-\left(\tilde{\rho}_{i}^{B}\right)^{2}\right\} \frac{\kappa_{i}^{A}}{\gamma \sigma_{i}^{A}} x+\tilde{\rho}_{i}^{A} \tilde{\rho}_{i}^{B} \frac{\kappa_{i}^{B}}{\gamma \sigma_{i}^{A}} x-\tilde{\rho}_{i}^{A} \frac{\kappa_{i}^{M}}{\gamma \sigma_{i}^{A}} x\right] /\left\{1-\left(\tilde{\rho}_{i}^{A}\right)^{2}-\left(\tilde{\rho}_{i}^{B}\right)^{2}\right\},
\end{gathered}
$$

and

$$
\left(\pi_{t}^{B}\right)^{*}=\left[\left\{1-\left(\tilde{\rho}_{i}^{A}\right)^{2}\right\} \frac{\kappa_{i}^{B}}{\gamma \sigma_{i}^{B}} x+\tilde{\rho}_{i}^{A} \tilde{\rho}_{i}^{B} \frac{\kappa_{i}^{A}}{\gamma \sigma_{i}^{B}} x-\tilde{\rho}_{i}^{B} \frac{\kappa_{i}^{M}}{\gamma \sigma_{i}^{B}} x\right] /\left\{1-\left(\tilde{\rho}_{i}^{A}\right)^{2}-\left(\tilde{\rho}_{i}^{B}\right)^{2}\right\}
$$

The hedging demand against undiversified systematic risk is measured by the differences between optimal strategies given in (3) and (4), (5), (6). It is either increased or decreased depending upon the sign of correlations $\tilde{\rho}_{i}^{A}$ and $\tilde{\rho}_{i}^{B}$. Thus, an investor demands different risk premia for bearing diversified and undiversified risks.

When we consider the case in which systematic risk no longer exists, but dynamic 
and asymmetric correlations between industry portfolios exist, i.e., when $\tilde{\rho}_{i}^{k}=0(k \in$ $\{A, B\}, i \in\{0,1\}),\left|\rho_{i}\right|<1$, and $\rho_{i} \neq 0 \mathbb{4}^{4}$ the optimal investment strategy reduces to the following:

$\left(\pi_{t}^{M}\right)^{*}=\frac{\kappa_{i}^{M}}{\gamma \sigma_{i}^{M}} x, \quad\left(\pi_{t}^{A}\right)^{*}=\frac{1}{1-\left(\rho_{i}\right)^{2}}\left[\frac{\kappa_{i}^{A}}{\gamma \sigma_{i}^{A}}-\rho_{i} \frac{\kappa_{i}^{B}}{\gamma \sigma_{i}^{A}}\right] x$, and $\left(\pi_{t}^{B}\right)^{*}=\frac{1}{1-\left(\rho_{i}\right)^{2}}\left[\frac{\kappa_{i}^{B}}{\gamma \sigma_{i}^{B}}-\rho_{i} \frac{\kappa_{i}^{A}}{\gamma \sigma_{i}^{B}}\right] x$

In this case, an optimal portfolio comprised of industry stocks is affected by both Sharpe ratios $\kappa_{i}^{A}$ and $\kappa_{i}^{B}$. Compared to $(3)$, the correlations $\rho_{i}$ between industry stocks would be crucial in deriving the optimal portfolio.

\section{Quantitative Analysis}

In this section, we perform an extensive quantitative analysis to discuss various properties of analytically tractable optimal investment strategies.

Baseline Parameter Values. Our modeling focus is on the effects of regime-dependent asymmetric correlations, abstracting away complex issues of other parameters. In light of such an objective, we assume symmetric values for other parameters. Reflecting today's low interest rate environment, we set risk-free interest rate to $1 \%$, i.e., $r_{0}=r_{1}=0.01$. We set equity premium to $7 \%$, i.e., expected rates of returns on the public market portfolio are $\mu_{0}^{M}=\mu_{1}^{M}=0.08$. We set market volatility to $23.5 \%$, i.e., $\sigma_{0}^{M}=\sigma_{1}^{M}=0.235$. Industry

\footnotetext{
${ }^{4}$ By assuming $\tilde{\rho}_{i}^{k}=0(k \in\{A, B\}, i \in\{0,1\})$, industry portfolios do not have any exposure to systematic risk as industry beta becomes zero.
} 
$A$ stock is assumed to have lower expected return and volatility than Industry $B$ stock, i.e., expected rates of returns on Industry $A$ and $B$ stocks are $\mu_{0}^{A}=\mu_{1}^{A}=0.05$, and $\mu_{0}^{B}=\mu_{1}^{B}=0.11$, volatilities are $\sigma_{0}^{A}=\sigma_{1}^{A}=0.2$, and $\sigma_{0}^{B}=\sigma_{1}^{B}=0.25$. Following the parameter values of relative risk aversion and regime intensities in Jang et al. (2007), we fix $\gamma=\psi^{-1}=2, \lambda_{0}=0.2353$, and $\lambda_{1}=1.7391$.

Benchmark. We first consider the simplest possible situation in which the market portfolio, Industry A and Industry B stocks are all independent i.e., $\tilde{\rho}_{i}^{k}(k \in\{A, B\}, i \in\{0,1\})$ and $\rho_{i}$ are all zero. Then, the optimal portfolio proportions given in (3) are

$$
\left(\pi_{t}^{M}\right)^{*} / x=63.4 \%, \quad\left(\pi_{t}^{A}\right)^{*} / x=50.0 \%, \quad\left(\pi_{t}^{B}\right)^{*} / x=80.0 \% .
$$

An investor is willing to invest more in Industry B stock than in the market portfolio and Industry $A$ stock. In the spirit of Merton $(1969,1971)$, the investor is highly dependent on Sharpe ratios when investing in the stock market.

Effects of Systematic Risk. Now, if we consider the systematic risk of Industry A and Industry B stocks, i.e., $\left|\tilde{\rho}_{i}^{k}\right|<1(k \in\{A, B\}, i \in\{0,1\}), \tilde{\rho}_{i}^{k} \neq 0$, and $\rho_{i}=0$, the market portfolio and Industry A and Industry B stocks are not perfectly correlated. We set the correlations $\tilde{\rho}^{A}\left(\equiv \tilde{\rho}_{0}^{A}=\tilde{\rho}_{1}^{A}\right)$ and $\tilde{\rho}^{B}\left(\equiv \tilde{\rho}_{0}^{B}=\tilde{\rho}_{1}^{B}\right)$ across regime 0 ("Bull") and regime 1 ("bear") to 0.5 and 0.1 , respectively. Then the optimal portfolio proportions given in (4), 
(5), and (6) are

$$
\left(\pi_{t}^{M}\right)^{*} / x=45.4 \%, \quad\left(\pi_{t}^{A}\right)^{*} / x=23.3 \%, \quad\left(\pi_{t}^{B}\right)^{*} / x=75.7 \%
$$

As a result of undiversified systematic risk given by (1), the optimal risky investment will reduce significantly compared to the benchmark case. This implies that the benchmark case does make sense only if an investor should be compensated by no more than the undiversifed systematic risk premium.

\section{Effects of Dynamic and Asymmetric Correlations at Market Portfolio Level.}

Next, we consider the case in which industry stocks are mutually independent i.e., $\rho_{i}=0$ $(i \in\{0,1\})$, but they have dynamic and asymmetric correlations with the market portfolio according to a regime switching environment. In this quantitative analysis, an investor is assumed to infer the status of the current regime by observing correlations, which are the parameters that change across regimes. We average the correlations across regime 0 and regime 1 by using the average fraction of time spent in each regime. With the Poisson jump intensities $\lambda_{0}$ and $\lambda_{1}$, the regime 0 (or the Bull regime) lasts on average $1 / \lambda_{0}$ and the regime 1 (or the bear regime) lasts on average $1 / \lambda_{1}$. Thus, the average fraction of time spent in the regime 0 and the regime 1 are given by

$$
\frac{1 / \lambda_{0}}{1 / \lambda_{0}+1 / \lambda_{1}}=\frac{\lambda_{1}}{\lambda_{0}+\lambda_{1}}
$$


and

$$
\frac{1 / \lambda_{1}}{1 / \lambda_{0}+1 / \lambda_{1}}=\frac{\lambda_{0}}{\lambda_{0}+\lambda_{1}}
$$

respectively. The average correlation $\tilde{\rho}^{k}(k \in\{A, B\})$ is then

$$
\tilde{\rho}^{k}=\frac{\lambda_{1}}{\lambda_{0}+\lambda_{1}} \tilde{\rho}_{0}^{k}+\frac{\lambda_{0}}{\lambda_{0}+\lambda_{1}} \tilde{\rho}_{1}^{k}
$$

The parameter values reflecting the case of dynamic and asymmetric correlations with the market portfolio can be chosen in the following two steps. First, correlations $\tilde{\rho}^{A}$ and $\tilde{\rho}^{B}$ across regime 0 ("Bull") and regime 1 ("bear") are set to 0.5 and 0.1 , respectively. Second, we obtain the values of $\tilde{\rho}_{1}^{A}$ using the relationship 77 by varying values of $\tilde{\rho}_{0}^{A}$ from 0.44 to 0.56 at intervals of 0.01 . The correlation pairs $\left(\tilde{\rho}_{0}^{A}, \tilde{\rho}_{1}^{A}\right)$ range from $(0.44,0.94)$ to $(0.56,0.06)$. Similarly, we also obtain the correlation pairs $\left(\tilde{\rho}_{0}^{B}, \tilde{\rho}_{1}^{B}\right)$ by changing values of $\tilde{\rho}_{0}^{B}$ from 0.04 to 0.16 at intervals of 0.01 .

The optimal portfolio proportions are adjusted according to the perturbations from above and below the baseline parameter values of correlation pairs $\left(\tilde{\rho}_{0}^{k}, \tilde{\rho}_{1}^{k}\right)$ for industry $k \in\{A, B\}$. The asymmetric correlation pairs result in asymmetric values of optimal portfolio proportions in regime 0 ("Bull") and regime 1 ("bear"), which are derived from (4), (5), and (6). For details, see Table C.3 ${ }^{5}$

\section{[Insert Table C.3 here.]}

\footnotetext{
${ }^{5}$ In Appendix, we also provide the portfolio proportions in regime 0 and regime 1 , and the weighted average of portfolio proportions across both regimes (Table C.6.
} 
Interestingly, we find that when the current level of correlation pairs $\left(\tilde{\rho}_{0}^{k}, \tilde{\rho}_{1}^{k}\right)$ is relatively high as in the Industry A case and when the sum of correlation pairs drift up from the baseline parameters, portfolio proportions $\left(\left(\pi_{t}^{M}\right)^{*} / x,\left(\pi_{t}^{A}\right)^{*} / x,\left(\pi_{t}^{B}\right)^{*} / x\right)$ produce radical changes. While when the sum of correlation pairs linger below the baseline values, portfolio proportions exhibit negligible changes. In contrast, when the current level of the correlation pairs is low as in the Industry B case, portfolio proportions hardly change for every correlation pairs.

\section{Effects of Dynamic and Asymmetric Correlations at Industry Portfolio Level.}

We allow for the case where there exist dynamic and asymmetric correlations between industry portfolios. We assume that the correlation between market portfolio and Industry stocks is zero, i.e., $\tilde{\rho}_{i}^{k}=0$ for Industry $k \in\{A, B\}$ in regime $i \in\{0,1\}$, but the correlations between industry stocks are nonzero, i.e., $\left|\rho_{i}\right|<1$ and $\rho_{i} \neq 0$.

The parameter values reflecting this case can be chosen as follows. First, we set the correlation $\rho$ across regime 0 ("Bull") and regime 1 ("bear") to 0.3 . Second, we use the relationship between correlations in regime 0 and regime 1 :

$$
\rho=\frac{\lambda_{1}}{\lambda_{0}+\lambda_{1}} \rho_{0}+\frac{\lambda_{0}}{\lambda_{0}+\lambda_{1}} \rho_{1}
$$

which is the weighted average of correlations $\rho_{0}$ and $\rho_{1}$ by the average fraction of time spent in regime $i(i \in\{0,1\})$. Third, we vary the values of $\rho_{0}$ from 0.21 to 0.39 at intervals of 0.02 , then the values of $\rho_{1}$ are determined by the relationship (8). The correlation pairs 
$\left(\rho_{0}, \rho_{1}\right)$ range from $(0.21,0.97)$ to $(0.39,-0.37)$ as shown in Table C.4

\section{[Insert Table C.4 here.]}

We emphasize that a similar pattern emerges to that in Table C.3. That is, when correlation pairs $\left(\rho_{0}, \rho_{1}\right)$ drift up from the baseline parameters, portfolio proportions,

$$
\left(\left(\pi_{t}^{M}\right)^{*} / x,\left(\pi_{t}^{A}\right)^{*} / x,\left(\pi_{t}^{B}\right)^{*} / x\right)
$$

yield substantial changes, whereas when the correlation pairs submerge below the baseline values, portfolio proportions exhibit small changes. The result is persistent for different combinations of the current level of correlation pairs $\left(\tilde{\rho}_{0}^{k}, \tilde{\rho}_{1}^{k}\right)$. Interestingly, asymmetric effects show jump-type movements in both cases when the correlation pairs increase from the baseline parameter values, which is hardly observed in Table C.3.

Sensitivity Analysis. We check in three ways the robustness of our quantitative results obtained so far according to the changes in the baseline parameter values. In our sensitivity analysis, we focus on the effects of dynamic and asymmetric correlations at the industry portfolio level.

First, we consider more conservative equity premia of Industry A and B stocks than the baseline equity premia. We set expected rates of returns on Industry A and B stocks to $4 \%$ and $10 \%$ rather than $5 \%$ and $11 \%$, i.e., $\mu_{0}^{A}=\mu_{1}^{A}=0.04$ and $\mu_{0}^{B}=\mu_{1}^{B}=0.10$. Second,

\footnotetext{
${ }^{6}$ In Appendix, we also provide the portfolio proportions in regime 0 and regime 1 , and the weighted average of portfolio proportions across both regimes (Table C.7).
} 
we consider more volatile Industry A and B stocks than the baseline volatilities. We set volatilities of Industry A and B stocks to $21 \%$ and $26 \%$ rather than $20 \%$ and $25 \%$, i.e., $\sigma_{0}^{A}=\sigma_{1}^{A}=0.21$ and $\sigma_{0}^{B}=\sigma_{1}^{B}=0.26$. Finally, we consider a more risk-averse individual by setting her risk aversion to 3 rather than 2 , i.e., $\gamma=3$.

Table C.5 reports the results of our sensitivity analysis. In the table, each column on the right panel shows the results of portfolio proportions according to the changes in the baseline parameter values stated above. For a fixed correlation pair, the portfolio proportions are lower if the investment opportunity worsens as compared to those under the baseline parameter values (Table C.4), i.e., when the expected returns on industry stocks are lower or their volatilities are higher, or both. Increased risk aversion leads the investor to take less risk on her risky investment in industry stocks. For various correlation pairs, our sensitivity analysis confirms that there are quantitative differences according to the changes of the parameter values, but the qualitative features of the effects of dynamic and asymmetric correlations at the industry portfolio level are preserved. We can still observe that portfolio proportions are inclined to be adjusted very much as the values of correlation pair increase from the baseline value, while they tend to be relatively consistent as the correlation values decrease from the baseline value.

\section{Empirical Application}

Data Description. For the empirical analysis of time-varying asymmetric correlation between the S\&P 500 and five industries (consumer, manufacture, hi-tech, health, and others) and efficient frontiers with dynamic \& asymmetric correlations, we have used the 
monthly data of industries from July 1990 to July 2015 by using Fama-French data library (Figure C.1. Figure C.2. . For obtaining regime-dependent time-varying correlations of industry portfolios and filtered probabilities for two regimes, we have used the monthly data of consumer durables and non-durables sectors from July 1926 to December 2015 by using Fama-French data library. Finally, for the test of empirical performance of various investment strategies, we have calculated the final wealth paths with a focus on the global financial crisis period (from March 2007 to June 2009) using those strategies including ours and the four performance measures (cumulative return, standard deviation, Sharpe ratio, and maximum drawdown).

Empirical Analysis Design. We further test whether our theoretical derivations yield meaningful investment performance. Amalgamating sector returns with feasible trading strategies is not an easy task. To do so, we provide empirical evidence supporting the use of regime-dependent correlation to manage portfolios using two industry portfolios. Following Gomes et al. (2009), we consider a dynamic production economy with a nondurable-good industry and a durable-good industry 7 Based on implications from our model solution, we then calculate the optimal risky weights for durable sector A and non-durable sector B. Then, we examine the empirical performance of our proposed strategy compared to other heuristic portfolios such as $1 / \mathrm{n}$, maximum diversification, inverse volatility, equal

\footnotetext{
${ }^{7}$ Given that the demand for non-durable goods and services is less cyclical than that for durable goods, we are likely to capture significant investment performance by adopting our regime-dependent investment strategies.
} 
risk parity, and two tail-risk parity strategies. Note that the optimal risky weights for sectors $\mathrm{A}$ and $\mathrm{B}$ are adjusted by the regime-dependent correlation $\rho_{i}$. For the detailed algorithms of parameter estimation, refer to Appendix B.

Specifically, we construct our portfolio based on our derived equations 5 and 6 in which we only consider systematic risk part. That is, the amount of investment in the Industry $A$ stock is adjusted by Sharpe ratio $\kappa_{i}^{B}$ for Industry $B$ stock due to its hedging effect against negative outcomes of Industry $A$ stock. We select consumer durables and non-durables as our test industry portfolios following Gomes et al. (2009). Consumer durables consists of furniture and household equipment, motor vehicles and parts, and other durable goods. Nondurable goods consists of clothing and shoes, food, fuel oil and coal, gasoline and oil, and other nondurable goods.

To construct real-time portfolios with given information, one iteration of the rolling sample approach has several steps. Starting at time $t=M$, the parameters required for each of the portfolio strategies over the estimation window of the $\mathrm{M}$ previous months are estimated. For example, this step entails estimating the sample volatility and variancecovariance matrix and relevant correlations on excess returns over the previous 36 months when $\mathrm{M}=36$. The next step involves solving the constrained optimization problem for each of the portfolio strategies. The final step is to compute the portfolio return in period t, based on the optimal set of asset weights at time t-1. In the first iteration, the portfolio's return would be the excess returns on risky assets at time $\mathrm{t}=60$ with asset weights $\mathrm{t}=$ 59 when $\mathrm{M}=60$. This rolling window approach involves adding the return for the next month in the data set and dropping the earliest return, which keeps the estimation window 
length fixed.

Empirical Results. The two panels in Figure C.3 exhibit the main estimation results of the regime-dependent dynamic correlations and the filtered probabilities for two distinctive regimes, respectively. The former panel confirms that correlations between consumer durables and non-durables tend to increase in highly volatile bear markets. The latter panel displays a higher probability of increased correlation during the Oil Shock period (1973-1974) as well as of decreased correlation during the Great Moderation period (from the mid-1980s to the financial crisis in 2007). Compared to the Great Depression (19291939) and the Oil Shock periods, surprisingly, the filtered probability of higher correlation status during the Global Financial Crisis period (March 2007 to June 2009) looks mild.

\section{[Insert Figure C.3 here.]}

We examine the empirical performance of our proposed strategy compared to other asset allocation strategies with a special focus on the Global Financial Crisis period (March 2007 to June 2009). 8 The performance is well confirmed by Figure C.4 in which the thick black line captures the final wealth path of our portfolio and other lines display the final wealth paths for corresponding heuristic portfolios and all portfolios that are constructed by using two reference indices, namely consumer durables and non-durables. Our proposed portfolio indicated by the thick black line outperforms other heuristic portfolios in terms of defending

\footnotetext{
${ }^{8}$ Our choice of the data period with a focus on the Global Financial Crisis (March 2007 to June 2009) is to investigate the empirical performance of our optimal strategies especially at times of economic recessions.
} 
the final wealth as well as four widely accepted measures including cumulative return, standard deviation, Sharpe ratio, and maximum drawdown during the Global Financial Crisis. One notable finding is that the final wealth path of the portfolio incorporating regime-dependent correlations dominated other final wealth paths for the whole period. The Value-at-Risk (VaR) based tail-risk parity portfolio seemed to work better only for the first half of the sample period with the lowest final wealth at the end of the cited period. Contrary to approximately $30 \%$ of maximum drawdown for the latter half period, our proposed portfolio confines its maximum loss to $10 \%$.

We have confirmed the superior empirical performance of our strategies to others when applied to the full sample data from January 1980 to July 2015 (Figure C.5). Interestingly, over the full sample data, we could also find that our proposed portfolio considering regime-dependent dynamic correlations outperforms, especially in the Global Financial Crisis period, others in the aspect of the final wealth. Our analysis therefore suggests that consideration of dynamic and asymmetric correlations between industry and market portfolios is an important factor in the attainment of successful investment return in the crisis period. In contrast to standard symmetric model, changes in the investment opportunities in one regime have an influence on the optimal investment strategy in another regime. Loosely speaking, the greater the change in the investment opportunity set after regime switching from the bull (bear) regime to the bear (bull) regime just as economic recessions, the greater the benefit of considering time-varying correlation dynamics. This inversely implies that misestimating or overlooking such a regime-dependent correlation can be costly to investors. For instance, if an investor underestimates the correlation and 
adopts the corresponding heuristic investment strategies under the wrong estimation, the expected wealth loss from these trading strategies would be very high.

\section{[Insert Figure C.4 here.]}

\section{Conclusion}

We develop a tractable investment model at industry portfolio level under dynamic and asymmetric correlations between portfolios. In our regime-dependent correlations environment, we derive in closed-form the optimal consumption and investment strategies. We find significant adjustments in the optimal investment portfolio, reflecting a compensation for the exposure to dynamic and asymmetric correlation. This implies that ignoring such time-varying and asymmetric correlations between portfolios can be costly to investors when applied to a construction of the optimal portfolio. Our empirical test of overall per-

formance of the model-based investment strategy shows that ours can outperform other heuristic strategies such as such as $1 / n$, maximum diversification, inverse volatility, equal risk parity, and two tail-risk parity strategies. 


\section{References}

[1] Ang, A., Bekaert, G. 2002. International Asset Allocation with Regime Shifts. Review of Financial Studies. 15, 1137-1187.

[2] Ball, C. A., Torous, W. N., 2000. Stochastic Correlation across International Stock Markets. Journal of Empirical Finance. 7, 373-388.

[3] Buraschi, A., Porchia, P., Trojani, F., 2010. Correlation Risk and Optimal Portfolio Choice. Journal of Finance. 65, 393-420.

[4] Cappiello, L., Engle, R. F., Sheppard, K. 2006. Asymmetric Dynamics in the Correlations of Global Equity and Bond Returns. Journal of Financial Economics. 2006. 25, $537-572$.

[5] Chava, S., Hsu, A., Zeng, L. 2018. Does History Repeat Itself? Business Cycle and Industry Returns. Georgia Tech Scheller College of Business Research Paper, No. 17-24.

[6] Cochrane, J. H. 2017. Macro-Finance. Review of Finance. 21, 945-985.

[7] Dai, M., Cai, J., Chen, X. 2018. Portfolio Selection with Capital Gains Tax, Recursive Utility, and Regime Switching. Management Science. 64, 2308-2324.

[8] Dai, M., Li, P.F., Liu, H., Wang, Y. 2016. Portfolio Choice with Market Closure and Implications for Liquidity Premia. Management Science. 62, 368-386. 
[9] Duffie, D., Epstein, L. G., 1992. Stochastic Differential Utility. Econometrica. 60, 353-394.

[10] Engle, R. 2002. Dynamic Conditional Correlation: A Simple Class of Multivariate Generalized Autoregressive Conditional Heteroskedasticity Models. Journal of Business \& Economic Statistics. 20, 339-350.

[11] Glosten, L. R., Jagannathan, R., Runkle, D. E. 1993. On the Relation between the Expected Value and the Volatility of the Nominal Excess Return on Stocks. Journal of Finance. 48, 1779-1801.

[12] Gomes, J. F., Kogan, L., Yogo, M. 2009. Durability of Output and Expected Stock Returns. Journal of Political Economy. 117, 941-986.

[13] Hamilton, J. D. 1989. A New Approach to the Economic Analysis of Nonstationary Time Series and the Business Cycle. Econometrica. 57, 357-384.

[14] Jang, B. G., Koo, H. K., Liu, H., Loewenstein, M. 2007. Liquidity Premia and Transaction Costs. Journal of Finance. 62, 2329-2366.

[15] Kalotychou, E., Staikouras S. K., Zhao G., 2014. The Role of Correlation Dynamics in Sector Allocation. Journal of Banking and Finance. 48, 1-12.

[16] Liu, H., Loewenstein, M. 2013. Market Crashes, Correlated Illiquidity, and Portfolio Choice. Management Science. 59, 715-732. 
[17] Markowitz, H. 1952. Portfolio Selection. Journal of Finance. 7, 77-91.

[18] Merton, R. C. 1969. Lifetime Portfolio Selection under Uncertainty: The ContinuousTime Case. Review of Economics and Statistics. 51, 247-257.

[19] Merton, R. C., 1971. Optimal Consumption and Portfolio Rules in a Continuous-Time Model. Journal of Economic Theory. 51, 373-413.

[20] Phylaktis, K., Xia, L., 2009. Equity Market Comovement and Contagion: A Sectoral Perspective. Financial Management. 38, 381-409. 


\section{Appendix A. Appendix}

The dynamic programming approach leads to the following system of Hamilton-JacobiBellman (HJB) equations for $i, j \in\{0,1\}$ (Merton, 1971):

$$
\begin{aligned}
0= & \max _{\left(c, \pi^{M}, \pi^{A}, \pi^{B}\right)}\left[f\left(c, V_{i}\right)+\left(r_{i} x-c\right) \frac{\partial V_{i}}{\partial x}+\frac{1}{2}\left(\pi^{M}\right)^{2}\left(\sigma_{i}^{M}\right)^{2} \frac{\partial^{2} V_{i}}{\partial x^{2}}+\pi^{M} \sigma_{i}^{M} \kappa_{i}^{M} \frac{\partial V_{i}}{\partial x}\right. \\
& +\frac{1}{2}\left(\pi^{A}\right)^{2}\left(\sigma_{i}^{A}\right)^{2} \frac{\partial^{2} V_{i}}{\partial x^{2}}+\pi^{A} \sigma_{i}^{A} \kappa_{i}^{A} \frac{\partial V_{i}}{\partial x}+\frac{1}{2}\left(\pi^{B}\right)^{2}\left(\sigma_{i}^{B}\right)^{2} \frac{\partial^{2} V_{i}}{\partial x^{2}}+\pi^{B} \sigma_{i}^{B} \kappa_{i}^{B} \frac{\partial V_{i}}{\partial x} \\
& \left.+\tilde{\rho}_{i}^{A} \pi^{M} \sigma_{i}^{M} \pi^{A} \sigma_{i}^{A} \frac{\partial^{2} V_{i}}{\partial x^{2}}+\tilde{\rho}_{i}^{B} \pi^{M} \sigma_{i}^{M} \pi^{B} \sigma_{i}^{B} \frac{\partial^{2} V_{i}}{\partial x^{2}}+\rho_{i} \pi^{A} \sigma_{i}^{A} \pi^{B} \sigma_{i}^{B} \frac{\partial^{2} V_{i}}{\partial x^{2}}+\lambda_{i}\left(V_{j}-V_{i}\right)\right]
\end{aligned}
$$

where $V_{i}(x)$ represents the value function with respect to an investor's initial wealth $x$ in regime $i$. The optimality conditions for consumption $c$ and stock holdings $\pi^{M}, \pi^{A}$, and $\pi^{B}$ follow

$$
\begin{aligned}
& c_{t}^{*}=\left\{\frac{1}{\rho^{*}}\left((1-\gamma) V_{i}\right)^{\theta-1} \frac{\partial V_{i}}{\partial x}\right\}^{-\psi}, \\
& \left(\pi_{t}^{M}\right)^{*}=-\frac{\kappa_{i}^{M}}{\sigma_{i}^{M}} \frac{\partial V_{i}}{\partial x} / \frac{\partial^{2} V_{i}}{\partial x^{2}}-\tilde{\rho}_{i}^{A} \frac{\sigma_{i}^{A}}{\sigma_{i}^{M}}\left(\pi_{t}^{A}\right)^{*}-\tilde{\rho}_{i}^{B} \frac{\sigma_{i}^{B}}{\sigma_{i}^{M}}\left(\pi_{t}^{B}\right)^{*}, \\
& \left(\pi_{t}^{A}\right)^{*}=-\frac{\kappa_{i}^{A}}{\sigma_{i}^{A}} \frac{\partial V_{i}}{\partial x} / \frac{\partial^{2} V_{i}}{\partial x^{2}}-\rho_{i} \frac{\sigma_{i}^{B}}{\sigma_{i}^{A}}\left(\pi^{B}\right)^{*}, \quad \text { and }, \\
& \left(\pi_{t}^{B}\right)^{*}=-\frac{\kappa_{i}^{B}}{\sigma_{i}^{B}} \frac{\partial V_{i}}{\partial x} / \frac{\partial^{2} V_{i}}{\partial x^{2}}-\rho_{i} \frac{\sigma_{i}^{A}}{\sigma_{i}^{B}}\left(\pi^{A}\right)^{*} .
\end{aligned}
$$

We conjecture the form of $V_{i}$ as the following: for any $x>0$,

$$
V_{i}(x)=K_{i} \frac{x^{1-\gamma}}{1-\gamma},
$$

where $K_{i}$ is a constant to be determined for $i \in\{0,1\}$. A straightforward calculation leads to optimal consumption and optimal stock holdings in the theorem in which $K_{i}$ is a solution to the following system of equations for $i, j \in\{0,1\}$ : for notational simplicity, we 
let

$$
\begin{aligned}
\operatorname{cons}^{M} \equiv\left[\left\{1-\left(\rho_{i}\right)^{2}\right\} \frac{\kappa_{i}^{M}}{\gamma \sigma_{i}^{M}}\right. & +\left(\tilde{\rho}_{i}^{A} \rho_{i} \frac{\sigma_{i}^{B}}{\sigma_{i}^{M}}-\tilde{\rho}_{i}^{B} \frac{\sigma_{i}^{B}}{\sigma_{i}^{M}}\right) \frac{\kappa_{i}^{B}}{\gamma \sigma_{i}^{B}} \\
& \left.+\left(\tilde{\rho}_{i}^{B} \rho_{i} \frac{\sigma_{i}^{A}}{\sigma_{i}^{M}}-\tilde{\rho}_{i}^{A} \frac{\sigma_{i}^{A}}{\sigma_{i}^{M}}\right) \frac{\kappa_{i}^{A}}{\gamma \sigma_{i}^{A}}\right] /\left\{1-\left(\rho_{i}\right)^{2}-\left(\tilde{\rho}_{i}^{A}\right)^{2}-\left(\tilde{\rho}_{i}^{B}\right)^{2}+2 \rho_{i} \tilde{\rho}_{i}^{A} \tilde{\rho}_{i}^{B}\right\},
\end{aligned}
$$

$$
\begin{aligned}
\operatorname{cons}^{A} \equiv\left[\left\{1-\left(\tilde{\rho}_{i}^{B}\right)^{2} \frac{\kappa_{i}^{A}}{\gamma \sigma_{i}^{A}}\right.\right. & +\left(\tilde{\rho}_{i}^{A} \tilde{\rho}_{i}^{B} \frac{\sigma_{i}^{B}}{\sigma_{i}^{A}}-\rho_{i} \frac{\sigma_{i}^{B}}{\sigma_{i}^{A}}\right) \frac{\kappa_{i}^{B}}{\gamma \sigma_{i}^{B}} \\
& \left.\left.+\left(\tilde{\rho}_{i}^{B} \rho_{i} \frac{\sigma_{i}^{M}}{\sigma_{i}^{A}}-\tilde{\rho}_{i}^{A} \frac{\sigma_{i}^{M}}{\sigma_{i}^{A}}\right) \frac{\kappa_{i}^{M}}{\gamma \sigma_{i}^{M}}\right\}\right] /\left\{1-\left(\rho_{i}\right)^{2}-\left(\tilde{\rho}_{i}^{A}\right)^{2}-\left(\tilde{\rho}_{i}^{B}\right)^{2}+2 \rho_{i} \tilde{\rho}_{i}^{A} \tilde{\rho}_{i}^{B}\right\},
\end{aligned}
$$

$$
\begin{aligned}
\operatorname{cons}^{B} \equiv\left[\left\{1-\left(\tilde{\rho}_{i}^{A}\right)^{2}\right\} \frac{\kappa_{i}^{B}}{\gamma \sigma_{i}^{B}}\right. & +\left(\tilde{\rho}_{i}^{A} \tilde{\rho}_{i}^{B} \frac{\sigma_{i}^{A}}{\sigma_{i}^{B}}-\rho_{i} \frac{\sigma_{i}^{A}}{\sigma_{i}^{B}}\right) \frac{\kappa_{i}^{A}}{\gamma \sigma_{i}^{A}} \\
& \left.+\left(\tilde{\rho}_{i}^{A} \rho_{i} \frac{\sigma_{i}^{M}}{\sigma_{i}^{B}}-\tilde{\rho}_{i}^{B} \frac{\sigma_{i}^{M}}{\sigma_{i}^{B}}\right) \frac{\kappa_{i}^{M}}{\gamma \sigma_{i}^{M}}\right] /\left\{1-\left(\rho_{i}\right)^{2}-\left(\tilde{\rho}_{i}^{A}\right)^{2}-\left(\tilde{\rho}_{i}^{B}\right)^{2}+2 \rho_{i} \tilde{\rho}_{i}^{A} \tilde{\rho}_{i}^{B}\right\},
\end{aligned}
$$

then

$$
\begin{aligned}
0= & \frac{\psi^{-1}}{1-\psi^{-1}}\left(\rho^{*}\right)^{\psi} K_{i}^{-\theta \psi}-\frac{\rho^{*}}{1-\psi^{-1}}+r_{i}-\frac{1}{2} \gamma\left(\sigma_{i}^{M}\right)^{2}\left(\operatorname{cons}^{M}\right)^{2}+\sigma_{i}^{M} \kappa_{i}^{M} \operatorname{cons}^{M} \\
& -\frac{1}{2} \gamma\left(\sigma_{i}^{A}\right)^{2}\left(\operatorname{cons}^{A}\right)^{2}+\sigma_{i}^{A} \kappa_{i}^{A} \operatorname{cons}^{A}-\frac{1}{2} \gamma\left(\sigma_{i}^{B}\right)^{2}\left(\operatorname{cons}^{B}\right)^{2}+\sigma_{i}^{B} \kappa_{i}^{B} \operatorname{cons}^{B}-\gamma \tilde{\rho}^{A} \sigma_{i}^{M} \sigma_{i}^{A} \operatorname{cons}^{M} \operatorname{cons}^{A} \\
& -\gamma \tilde{\rho}_{i}^{B} \sigma_{i}^{M} \sigma_{i}^{B} \operatorname{cons}^{M} \operatorname{cons}^{B}-\gamma \rho_{i} \sigma_{i}^{A} \sigma_{i}^{B} \operatorname{cons}^{A} \operatorname{cons}^{B}+\lambda_{i}\left(\frac{K_{j}}{K_{i}}-1\right) \frac{1}{1-\gamma} \text {. Q.E.D. }
\end{aligned}
$$




\section{Appendix B. Parameter Estimation Algorithm Details}

\section{GJR-GARCH Model Parameters}

Glosten, Jagannathan, and Runkle (1993, hereafter GJR) have proposed the GJRGARCH (Generalized Autoregressive Conditional Heteroskedasticity) as the following:

$$
\begin{gathered}
r_{t}=\mu+\epsilon_{t}, \\
\epsilon_{t}=\sigma_{t} z_{t}, z_{t} \sim \mathcal{N}\left(\mu, \sigma^{2}\right), \\
\sigma_{t}^{2}=\omega+\left(\alpha+\gamma I_{t-1}\right) \epsilon_{t-1}^{2}+\beta \sigma_{t-1}^{2}, \\
I_{t-1}= \begin{cases}0 & \text { if } r_{t-1} \geq \mu \\
1 & \text { if } r_{t-1}<\mu\end{cases}
\end{gathered}
$$

In the GJR-GARCH model, the market shock $\epsilon_{t-1}^{2}$ at time $t-1$ affects the volatility $\sigma_{t}$ at time $t$ via a non-linear relation represented by the following key parameters: $\alpha, \gamma, \omega$, and $\beta$. More specifically, the market shock $\epsilon_{t}$ at time $t$ responds to the positive shocks $(\alpha)$ and the negative shocks $(\gamma)$ in the stock market with the market shock $\epsilon_{t-1}$ at time $t-1$. In particular, when $\gamma>0$ such a response of the volatility to the market shocks becomes asymmetric. The other two parameters of $\omega$ and $\beta$ summarize respectively the volatility mean and the linear coefficient of a relation between $\sigma_{t}$ and $\sigma_{t-1}$. The following table shows our estimation results of the parameters of the GJR-GARCH model. 
Table B.1: GJR-GARCH Model Parameters

\begin{tabular}{lcccc}
\hline Index & $\omega$ & $\alpha$ & $\gamma$ & $\beta$ \\
\hline SP500 & 0.897 & 0.048 & 0.120 & 0.859 \\
Cnsmr & 0.923 & 0.044 & 0.095 & 0.880 \\
Manuf & 1.143 & 0.043 & 0.111 & 0.863 \\
HiTec & 1.384 & 0.057 & 0.158 & 0.831 \\
Hlth & 8.063 & 0.000 & 0.214 & 0.598 \\
Other & 1.886 & 0.022 & 0.176 & 0.845 \\
\hline
\end{tabular}

\section{DDC- and ADDC-GARCH Model Parameters}

The dynamic conditional correlation (DCC)-GARCH model by Engle (2002) differs from the GARCH model in that it incorporates dynamic and time-varying features of conditional correlation. Cappiello et al. (2006) have extended the DCC-GARCH to include asymmetric features of conditional correlation and named it as the ADCC-GARCH model. The detailed formulation of the ADCC-GARCH model is represented by the following equations:

$$
\begin{aligned}
& Q_{t}=\left(\bar{P}-a^{2} \bar{P}+b^{2} \bar{P}-g^{2} \bar{N}\right)+a^{2} \epsilon_{t-1} \epsilon_{t-1}^{\prime}+g^{2} n_{t-1} n_{t-1}^{\prime}+b^{2} Q_{t-1}, \\
& P_{t}=Q_{t}^{*-1} Q_{t} Q^{*-1}, \bar{P}=E\left[\epsilon_{t} \epsilon_{t}^{\prime}\right], n_{t}=I\left[\epsilon_{t}<0\right] \epsilon_{t}, \bar{N}=E\left[n_{t} n_{t}^{\prime}\right],
\end{aligned}
$$

where $P_{t}$ is the conditional correlation matrix of the standardized errors $\epsilon_{t}$ at time $t, \bar{P}=$ $\operatorname{cov}\left[\epsilon_{t} \epsilon_{t}^{\prime}\right]=E\left[\epsilon_{t} \epsilon_{t}^{\prime}\right]$ is the unconditional covariance matrix of $\epsilon_{t}$ at time $t, Q_{t}^{*}$ is the diagonal matrix with the square root of the diagonal elements of $Q_{t}$. Note that the covariance matrix $\bar{P}$ is positive definite when $a \geq 0, b \geq 0$, and $a+b<1$. Further, $Q_{t}$ is positive definite when $a+b+\delta g<1$, where $\delta$ is the maximum eigenvalue of $\bar{P}^{1 / 2} \bar{N} \bar{P} . P_{t}$ is then also positive definite. The ADDC-GARCH model reduces to the DCC-GARCH model when 
$g=0$. The following table shows our estimation results of the parameters of the DCCand ADCC-GARCH model.

Table B.2: DDC- and ADDC-GARCH Model Parameters

\begin{tabular}{lcc}
\hline Parameter & DDC Model & ADDC Model \\
\hline $\mathrm{a}$ & 0.053 & 0.041 \\
$\mathrm{~g}$ & & 0.046 \\
$\mathrm{~b}$ & 0.920 & 0.916 \\
\hline
\end{tabular}

\section{Regime Switching Model Parameters}

The two-state Markov chain regime switching model allows for the transition between bull and bear regimes, which captures an intrinsic property of the stock market with business cycles. For the dependent variable $y_{t}$, we consider two different states of $s: s=0$ (Bull) and $s=1$ (bear). Then,

$$
y_{s t}=\mu_{s}+\epsilon_{s t},
$$

where $\mu_{s}$ is the state-dependent mean of the dependent variable $y_{s t}$. The transition of states is stochastic and governed by the following transition probability matrix $P$ :

$$
P=\left[\begin{array}{ll}
p_{0,0} & p_{1,0} \\
p_{0,1} & p_{1,1}
\end{array}\right]=\left[\begin{array}{ll}
0.94 & 0.22 \\
0.06 & 0.78
\end{array}\right],
$$

where $p_{i, j}$ represents the probability of a switch from state $i$ to state $j$ for $i, j \in\{0,1\}$. 


\section{Appendix C. Figures and Tables}

Figure C.1: Time-varying asymmetric correlation between S\&P 500 and five industries

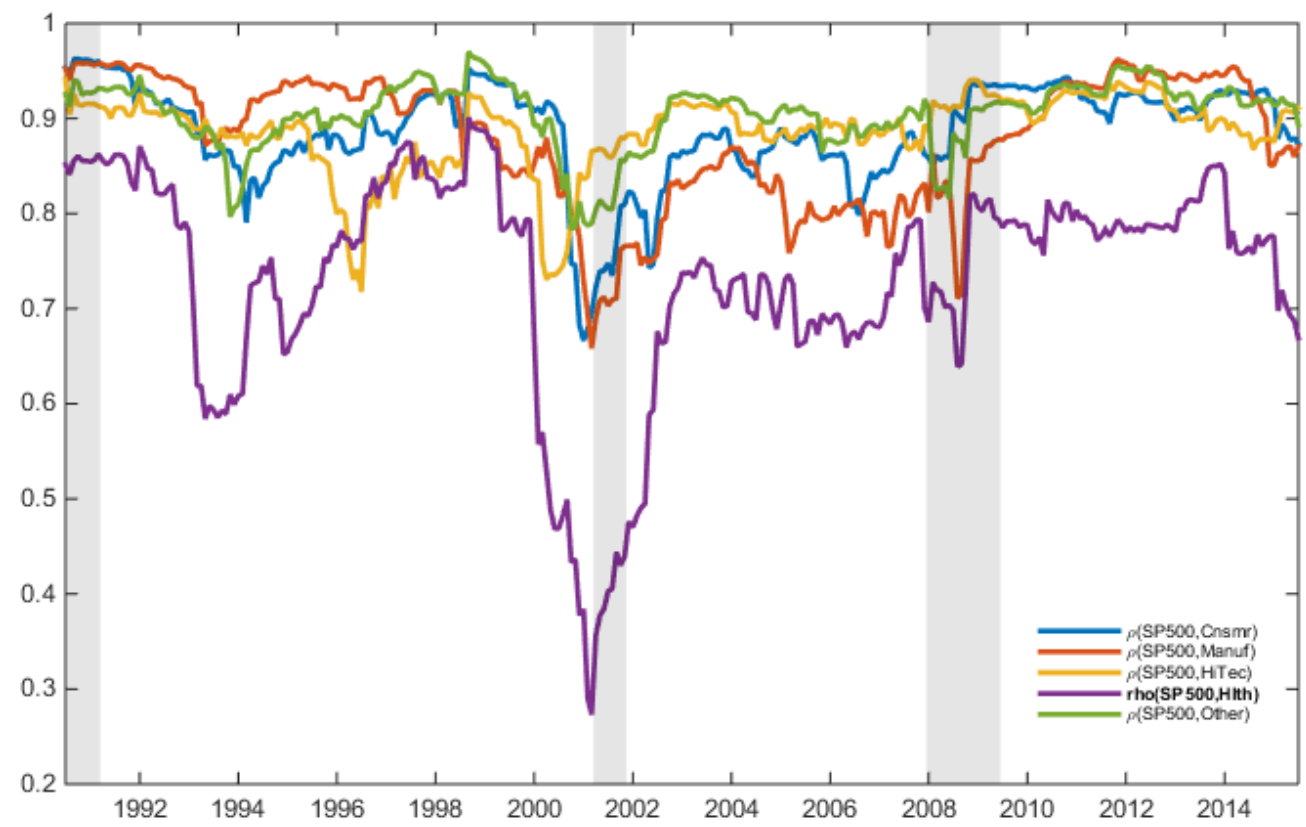

Note. This figure illustrates time-varying asymmetric correlation between the S\&P 500 and five industries. Data span from July 1990 to July 2015. Industry portfolios are obtained from FamaFrench data library and industries are consumer, manufacture, hi-tech, health and others. The shaded area represents recession as determined by the National Bureau of Economic Research (NBER). 
Figure C.2: Efficient frontiers with dynamic \& asymmetric correlations
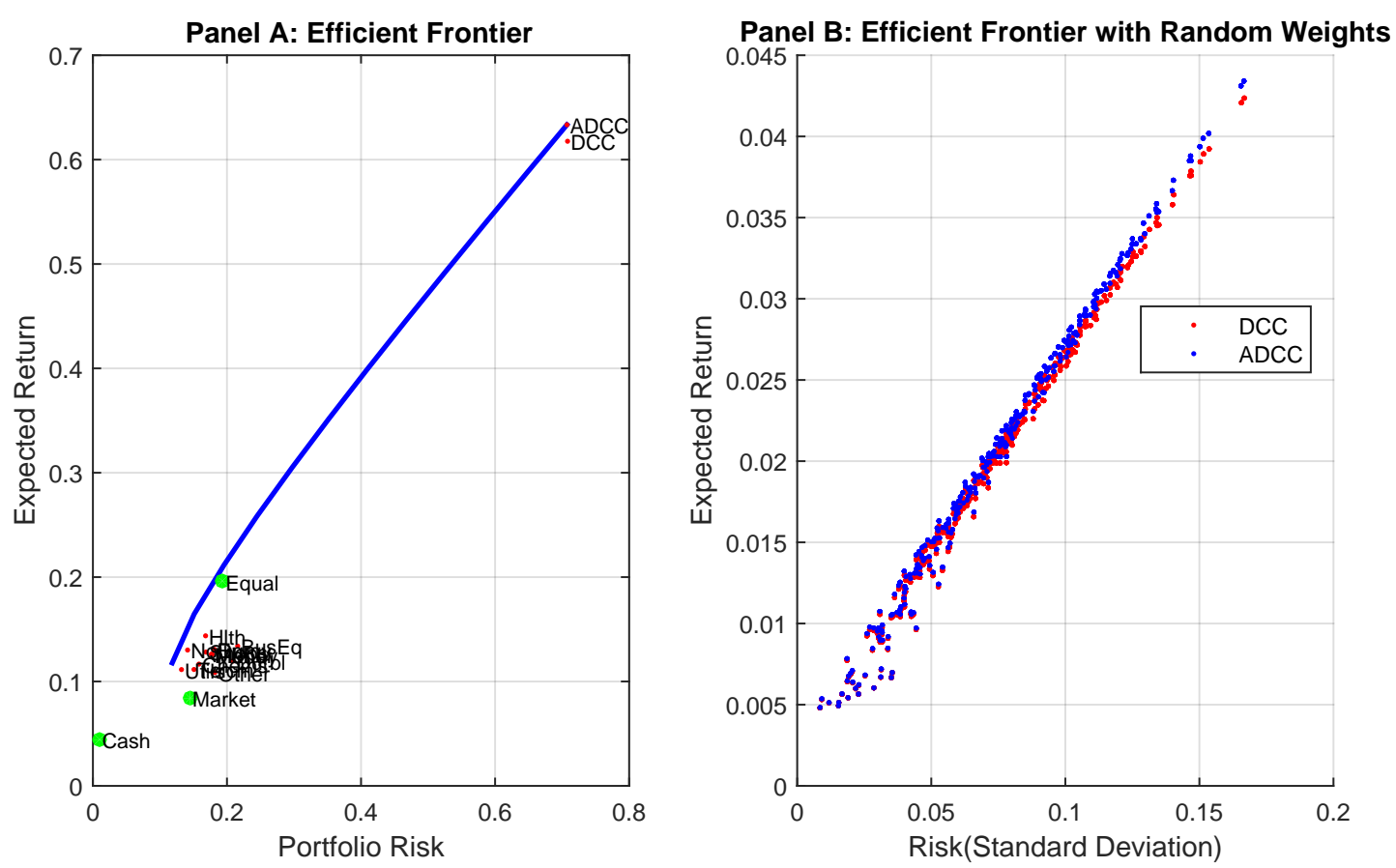

Note. This figure illustrates two efficient frontiers using 12 industry portfolios obtained from Fama-French data library. Data span from July 1950 to July 2015. Panel A depicts efficient frontier using 3 month T-bill proxy for risk-free rate, S\&P 500 for market return, 12 industry portfolios, and two aggregate industry portfolios constructed under the dynamic conditional correlation (DCC) and asymmetric DCC (ADCC) assumptions, respectively. Panel B exhibits an efficient frontier expansion after the asymmetric effect is employed to the forward-looking optimal risky asset weights. We plot the efficient frontier using the same risk-free rate, market return, and two aggregate industry portfolios as described in Panel A. Red dots represent the efficient frontier using a DCC aggregate industry portfolio and blue dots represent the efficient frontier using an ADCC aggregate industry portfolio. To capture the asymmetric effect, we apply 300 randomly generated weights. 
Figure C.3: Regime-dependent correlations and filtered probabilities

(a) Regime-dependent time-varying correlations

(a) Correlation $b / w$ Dur and NonDur in State 1

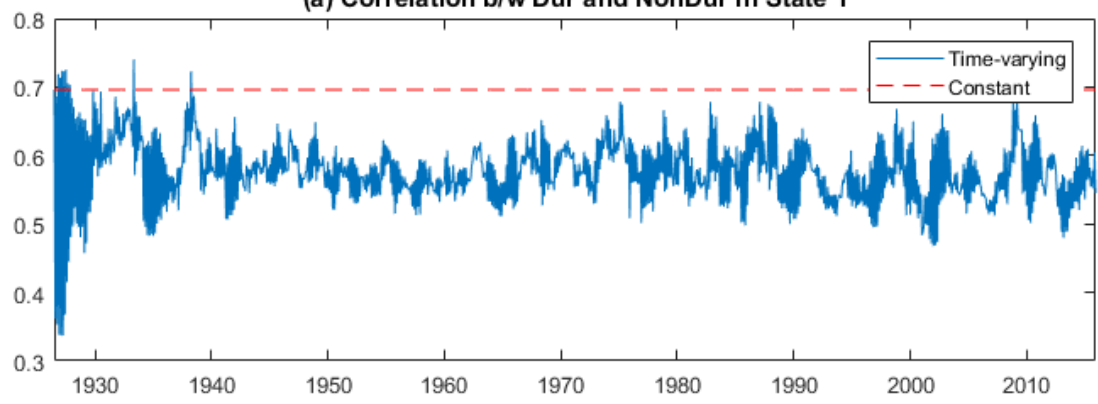

(a) Correlation b/w Dur and NonDur in State 2

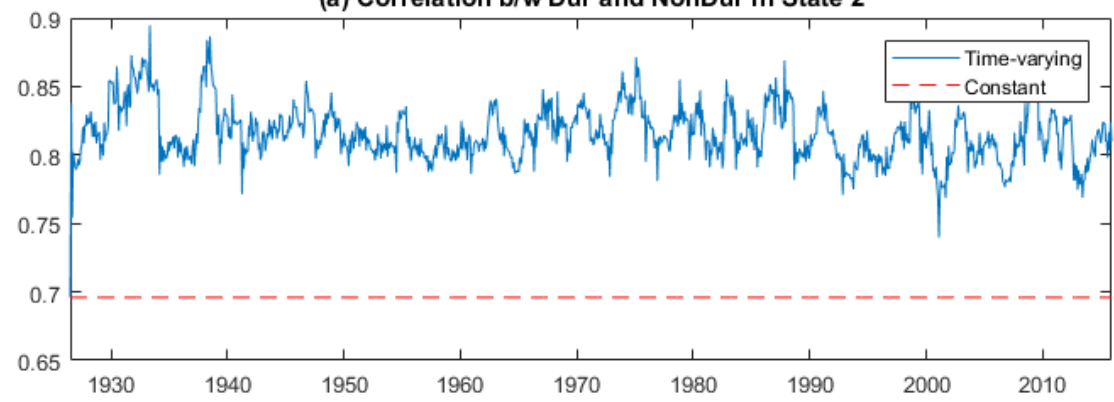

(b) Filtered probabilities for two regimes

(a) Filtered Prob of State1

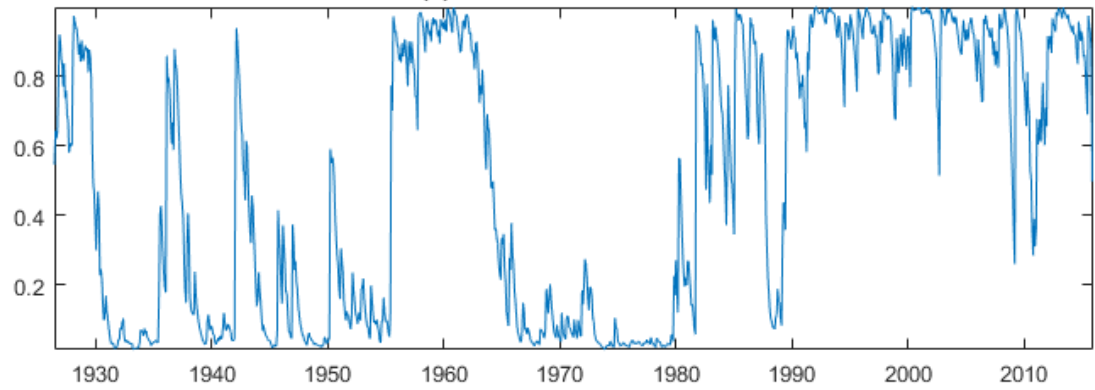

(b) Filtered Prob of State2

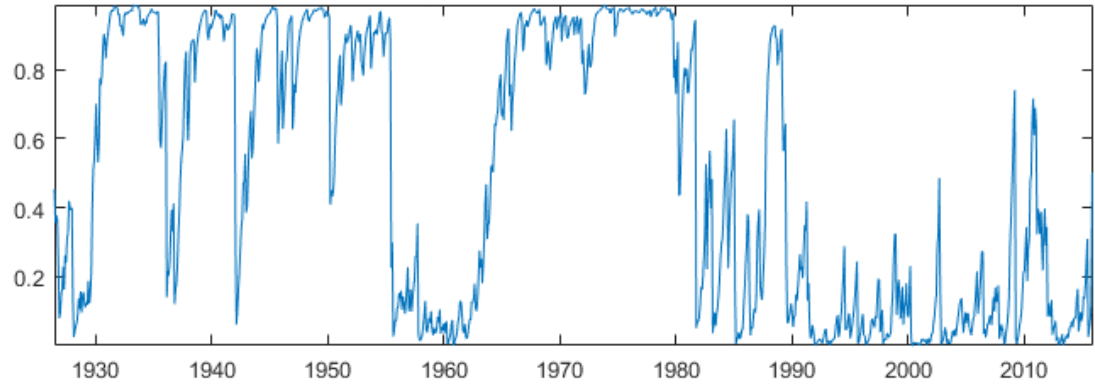

Note. Panel (a) depicts monthly time-series of dynamic correlations across different regimes from

July 1926 to December 2015. Panel (b) exhibits the corresponding filtered probabilities for the cited period. In this panel, State1 and State2 represent the bull market and the bear market, respectively. 
Figure C.4: Final wealth path and various performance measures

(a) Final wealth paths for various trading strategies

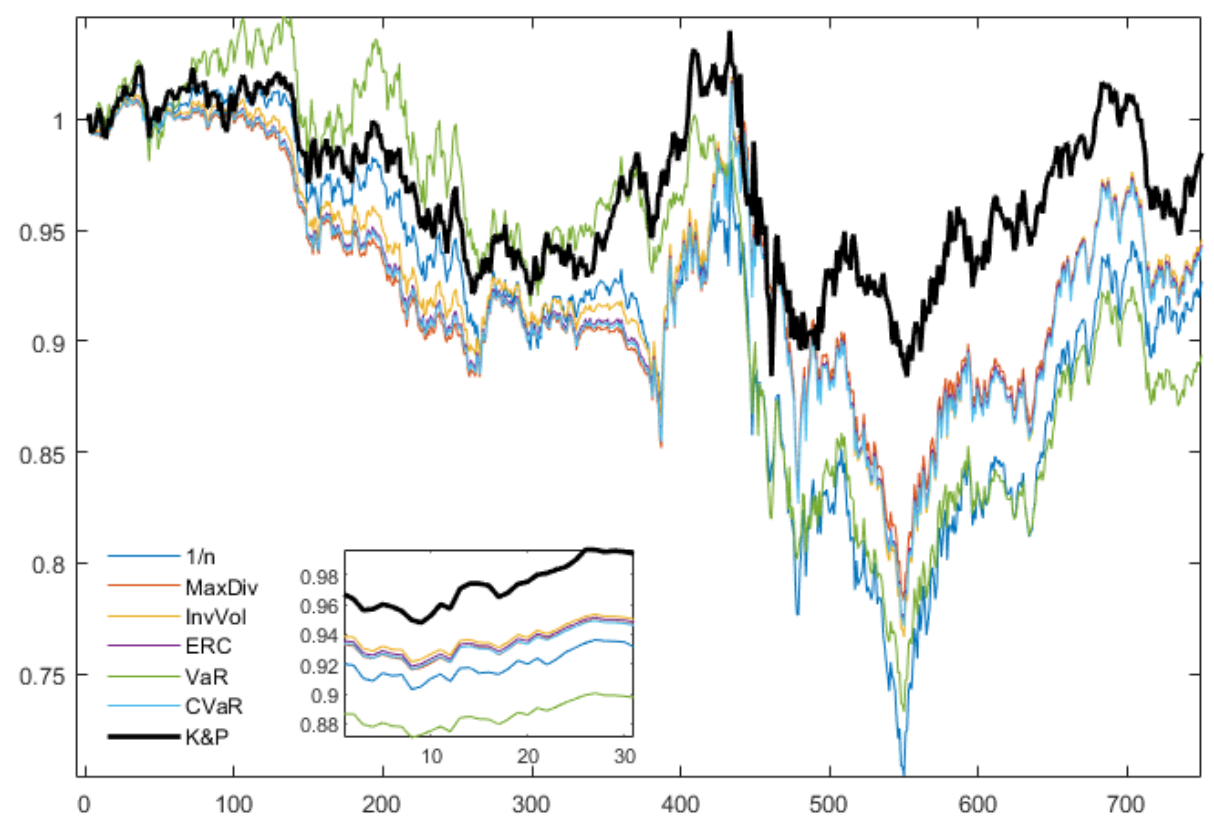

(b) Four performance measures
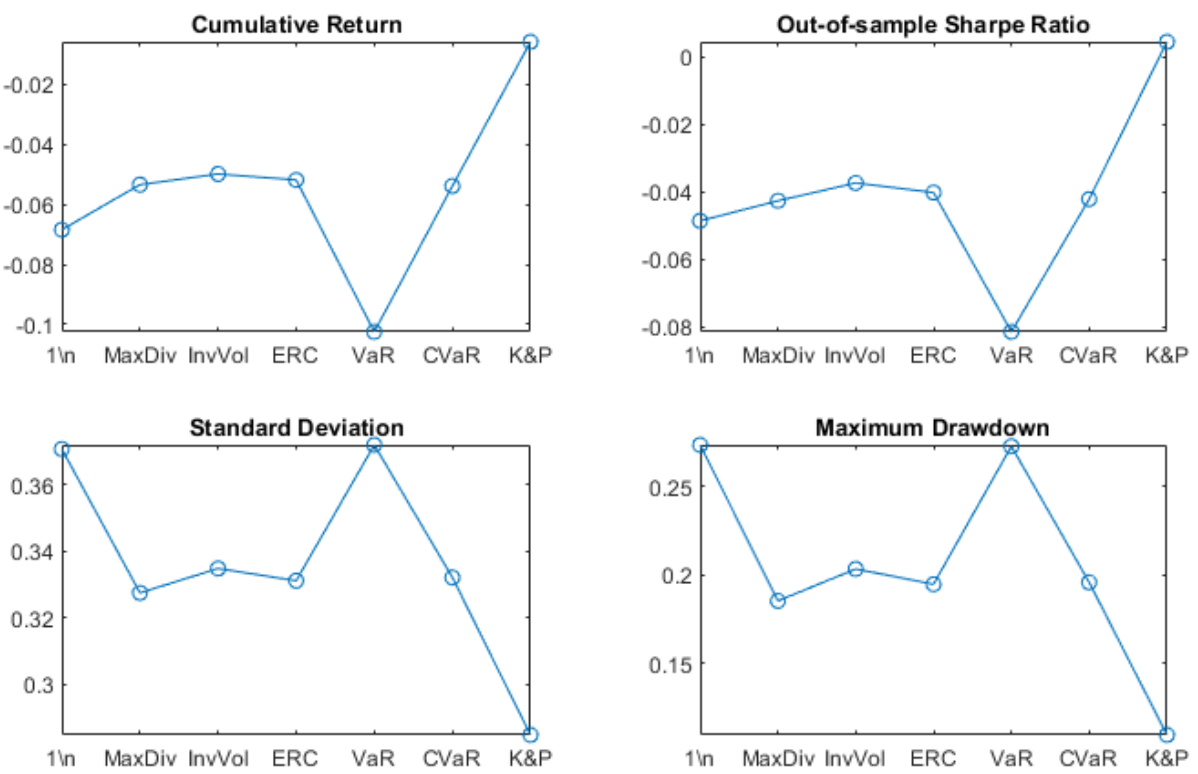

Note. Panel (a) displays the final wealth paths for various portfolios during the global financial crisis period, spanning March 2007 to June 2009. The thick black line (K\&P) captures the final wealth path of the proposed methodology. Panel (b) presents the four widely accepted performance measures including cumulative return, standard deviation, Sharpe ratio, and maximum drawdown. 
Figure C.5: Final wealth paths with the data period from January 1980 to July 2015

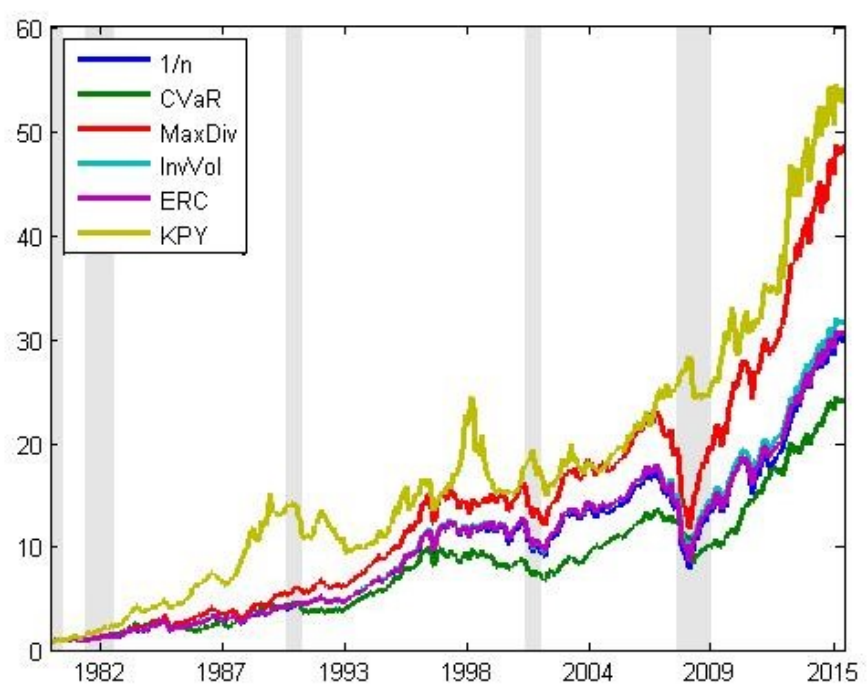

Note. This figure displays the final wealth paths for various portfolios with the data period from January 1980 to July 2015. 
Table C.3: Portfolio proportions $\left(\left(\pi_{t}^{M}\right)^{*} / x,\left(\pi_{t}^{A}\right)^{*} / x,\left(\pi_{t}^{B}\right)^{*} / x\right)$ according to correlation pairs

\begin{tabular}{|c|c|c|c|}
\hline \multicolumn{2}{|c|}{ 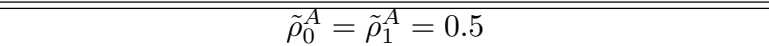 } & \multicolumn{2}{|c|}{ 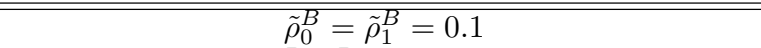 } \\
\hline Correlation Pairs $\left(\tilde{\rho}_{0}^{A}, \tilde{\rho}_{1}^{A}\right)$ & Portfolio Proportions & Correlation Pairs $\left(\tilde{\rho}_{0}^{B}, \tilde{\rho}_{1}^{B}\right)$ & Portfolio Proportions \\
\hline$(0.44,0.94)$ & $(57.5 \%, 9.9 \%, 74.6 \%)$ & $(0.04,0.54)$ & $(44.5 \%, 23.9 \%, 78.8 \%)$ \\
\hline$(0.45,0.87)$ & $(49.1 \%, 19.6 \%, 75.4 \%)$ & $(0.05,0.47)$ & $(45.1 \%, 23.5 \%, 77.7 \%)$ \\
\hline$(0.46,0.80)$ & $(47.0 \%, 21.9 \%, 75.6 \%)$ & $(0.06,0.40)$ & $(45.4 \%, 23.3 \%, 76.4 \%)$ \\
\hline$(0.47,0.72)$ & $(46.1 \%, 22.7 \%, 75.7 \%)$ & $(0.07,0.32)$ & $(45.4 \%, 23.3 \%, 76.4 \%)$ \\
\hline$(0.48,0.65)$ & $(45.6 \%, 23.1 \%, 75.7 \%)$ & $(0.08,0.25)$ & $(45.4 \%, 23.3 \%, 76.0 \%)$ \\
\hline$(0.49,0.57)$ & $(45.4 \%, 23.3 \%, 75.7 \%)$ & $(0.09,0.17)$ & $(45.4 \%, 23.3 \%, 75.8 \%)$ \\
\hline$(0.50,0.50)$ & $(45.4 \%, 23.3 \%, 75.7 \%)$ & $(0.10,0.10)$ & $(45.4 \%, 23.3 \%, 75.7 \%)$ \\
\hline$(0.51,0.43)$ & $(45.4 \%, 23.3 \%, 75.7 \%)$ & $(0.11,0.03)$ & $(45.4 \%, 23.3 \%, 75.8 \%)$ \\
\hline$(0.52,0.35)$ & $(45.6 \%, 23.2 \%, 75.7 \%)$ & $(0.12,-0.05)$ & $(45.5 \%, 23.2 \%, 76.0 \%)$ \\
\hline$(0.53,0.28)$ & $(45.8 \%, 23.2 \%, 75.7 \%)$ & $(0.13,-0.12)$ & $(45.8 \%, 23.1 \%, 76.4 \%)$ \\
\hline$(0.54,0.20)$ & $(46.0 \%, 23.1 \%, 75.7 \%)$ & $(0.14,-0.20)$ & $(46.2 \%, 22.8 \%, 77.0 \%)$ \\
\hline$(0.55,0.13)$ & $(46.3 \%, 23.0 \%, 75.6 \%)$ & $(0.15,-0.27)$ & $(47.0 \%, 22.4 \%, 77.9 \%)$ \\
\hline$(0.56,0.06)$ & $(46.8 \%, 23.0 \%, 75.6 \%)$ & $(0.16,-0.34)$ & $(48.1 \%, 21.8 \%, 79.1 \%)$ \\
\hline
\end{tabular}

Note. In the table, portfolio proportions denote the weighted average of portfolio proportions in regime 0 and regime 1 by the average fraction of time spent in regime 0 and regime 1 . The portfolio proportions given by bold type represent the portfolio proportions for the baseline parameter values. The baseline parameter values are as follows: risk-free interest rate $r_{0}=r_{1}=0.01$, expected rate of stock returns $\mu_{0}^{M}=\mu_{1}^{M}=0.08, \mu_{0}^{A}=\mu_{1}^{B}=0.05$, and $\mu_{0}^{B}=\mu_{1}^{B}=0.11$, stock volatilities $\sigma_{0}^{M}=\sigma_{1}^{M}=0.235$, $\sigma_{0}^{A}=\sigma_{1}^{A}=0.2$, and $\sigma_{0}^{B}=\sigma_{1}^{B}=0.25$, relative risk aversion $\gamma=2$, regime intensities $\lambda_{0}=0.2353$ and $\lambda_{1}=1.7391$, and correlation between industry stocks $\rho_{i}=0$ for regime $i \in\{0,1\}$. 
Table C.4: Portfolio proportions $\left(\left(\pi_{t}^{A}\right)^{*} / x,\left(\pi_{t}^{B}\right)^{*} / x\right)$ according to correlation pairs $\left(\rho_{0}, \rho_{1}\right)$

\begin{tabular}{|c|c|c|}
\hline $\begin{array}{c}\left(\rho_{0}, \rho_{1}\right) \\
\text { Correlation Pairs }\end{array}$ & $\begin{array}{c}\tilde{\rho}_{i}^{A}=0 \& \tilde{\rho}_{i}^{B}=0 \\
\text { Portfolio Proportions }\end{array}$ & $\begin{array}{l}\tilde{\rho}_{i}^{A}=0.5 \& \tilde{\rho}_{i}^{B}=0.1 \\
\text { Portfolio Proportions }\end{array}$ \\
\hline$(0.21,0.97)$ & $(-54.3 \%, 138.1 \%)$ & $(87.7 \%, 7.8 \%)$ \\
\hline$(0.23,0.82)$ & $(13.7 \%, 82.8 \%)$ & $(87.7 \%, 7.8 \%)$ \\
\hline$(0.25,0.67)$ & $(19.8 \%, 77.3 \%)$ & $(-22.2 \%, 113.9 \%)$ \\
\hline$(0.27,0.52)$ & $(21.5 \%, 75.4 \%)$ & $(-16.9 \%, 108.0 \%)$ \\
\hline$(0.29,0.37)$ & $(21.9 \%, 74.8 \%)$ & $(-15.6 \%, 106.3 \%)$ \\
\hline$(0.30,0.30)$ & $(22.0 \%, 74.7 \%)$ & $(-15.4 \%, 106.1 \%)$ \\
\hline$(0.31,0.23)$ & $(22.0 \%, 74.8 \%)$ & $(-15.5 \%, 106.3 \%)$ \\
\hline$(0.33,0.08)$ & $(21.9 \%, 75.2 \%)$ & $(-16.0 \%, 107.3 \%)$ \\
\hline$(0.35,-0.07)$ & $(21.9 \%, 76.2 \%)$ & $(-16.5 \%, 109.2 \%)$ \\
\hline$(0.37,-0.22)$ & $(22.2 \%, 77.6 \%)$ & $(-16.7 \%, 112.1 \%)$ \\
\hline$(0.39,-0.37)$ & $(23.3 \%, 79.9 \%)$ & $(-15.8 \%, 116.69 \%)$ \\
\hline
\end{tabular}

Note. In the table, portfolio proportions denote the weighted average of portfolio proportions in regime 0 and regime 1 by the average fraction of time spent in regime 0 and regime 1 . The portfolio proportions given by the bold type represent the portfolio proportions for the baseline parameter values. The baseline parameter values are as follows: risk-free interest rate $r_{0}=r_{1}=0.01$, expected rate of stock returns $\mu_{0}^{M}=\mu_{1}^{M}=0.08, \mu_{0}^{A}=\mu_{1}^{B}=0.05$, and $\mu_{0}^{B}=\mu_{1}^{B}=0.11$, stock volatilities $\sigma_{0}^{M}=\sigma_{1}^{M}=0.235$, $\sigma_{0}^{A}=\sigma_{1}^{A}=0.2$, and $\sigma_{0}^{B}=\sigma_{1}^{B}=0.25$, relative risk aversion $\gamma=2$, regime intensities $\lambda_{0}=0.2353$ and $\lambda_{1}=1.7391$, and correlation between Industry stocks $\rho_{i}=0.3$ for regime $i \in\{0,1\}$.

Table C.5: Portfolio proportions $\left(\left(\pi_{t}^{A}\right)^{*} / x,\left(\pi_{t}^{B}\right)^{*} / x\right)$ according to changes in baseline parameter values

\begin{tabular}{c||ccc}
\hline \hline$\left(\rho_{0}, \rho_{1}\right)$ & $\mu_{0}^{A}=\mu_{1}^{A}=0.04$ & $\sigma_{0}^{A}=\sigma_{1}^{A}=0.21$ & $\gamma=3$ \\
& $\mu_{0}^{B}=\mu_{1}^{B}=0.10$ & $\sigma_{0}^{B}=\sigma_{1}^{B}=0.26$ & \\
Correlation Pairs & Portfolio Proportions & Portfolio Proportions & Portfolio Proportions \\
\hline$(0.21,0.97)$ & $(-69.8 \%, 135.5 \%)$ & $(-50.9 \%, 128.3 \%)$ & $(-36.2 \%, 92.1 \%)$ \\
$(0.23,0.82)$ & $(2.70 \%, 77.6 \%)$ & $(12.0 \%, 76.8 \%)$ & $(9.10 \%, 55.2 \%)$ \\
$(0.25,0.67)$ & $(9.20 \%, 71.8 \%)$ & $(17.7 \%, 71.6 \%)$ & $(13.2 \%, 51.5 \%)$ \\
$(0.27,0.52)$ & $(11.0 \%, 69.9 \%)$ & $(19.2 \%, 69.9 \%)$ & $(14.3 \%, 50.3 \%)$ \\
$(0.29,0.37)$ & $(11.5 \%, 69.3 \%)$ & $(19.6 \%, 69.3 \%)$ & $(14.6 \%, 49.9 \%)$ \\
$(\mathbf{0 . 3 0 , 0 . 3 0 )}$ & $(\mathbf{1 1 . 5 \%}, \mathbf{6 9 . 2 \%})$ & $\mathbf{( 1 9 . 6 \% , 6 9 . 2 \% )}$ & $\mathbf{( 1 4 . 7 \% , 4 9 . 8 \% )}$ \\
$(0.31,0.23)$ & $(11.5 \%, 69.3 \%)$ & $(19.6 \%, 69.3 \%)$ & $(14.6 \%, 49.9 \%)$ \\
$(0.33,0.08)$ & $(11.4 \%, 69.7 \%)$ & $(19.5 \%, 69.7 \%)$ & $(14.6 \%, 50.2 \%)$ \\
$(0.35,-0.07)$ & $(11.3 \%, 70.6 \%)$ & $(19.5 \%, 70.5 \%)$ & $(14.6 \%, 50.8 \%)$ \\
$(0.37,-0.22)$ & $(11.4 \%, 72.0 \%)$ & $(19.9 \%, 71.9 \%)$ & $(14.8 \%, 51.8 \%)$ \\
$(0.39,-0.37)$ & $(12.2 \%, 74.0 \%)$ & $(20.8 \%, 74.0 \%)$ & $(15.5 \%, 53.3 \%)$ \\
\hline \hline
\end{tabular}

Note. In the table, portfolio proportions denote the weighted average of portfolio proportions in regime 0 and regime 1 by the average fraction of time spent in regime 0 and regime 1 . The portfolio proportions given by the bold type represent the portfolio proportions for the baseline parameter values. The baseline parameter values are as follows: risk-free interest rate $r_{0}=r_{1}=0.01$, expected rate of stock returns $\mu_{0}^{M}=\mu_{1}^{M}=0.08, \mu_{0}^{A}=\mu_{1}^{B}=0.05$, and $\mu_{0}^{B}=\mu_{1}^{B}=0.11$, stock volatilities $\sigma_{0}^{M}=\sigma_{1}^{M}=0.235$, $\sigma_{0}^{A}=\sigma_{1}^{A}=0.2$, and $\sigma_{0}^{B}=\sigma_{1}^{B}=0.25$, relative risk aversion $\gamma=2$, regime intensities $\lambda_{0}=0.2353$ and $\lambda_{1}=1.7391$, and correlation between Industry stocks $\rho_{i}=0.3$ for regime $i \in\{0,1\}$. 
Table C.6: Table: Portfolio proportions according to correlation pairs.

\begin{tabular}{cccc}
\hline \hline Correlation Pairs & Portfolio Proportions (Regime 0) & Portfolio Proportions (Regime 1) & Weighted Average Portfolio Proportions \\
\hline$(0.44,0.94)$ & $(45.4 \%, 26.5 \%, 75.7 \%)$ & $(147.4 \%,-113.4 \%, 66.1 \%)$ & $(57.5 \%, 9.9 \%, 74.6 \%)$ \\
$(0.45,0.87)$ & $(45.4 \%, 26.0 \%, 75.7 \%)$ & $(76.4 \%,-28.0 \%, 72.8 \%)$ & $(49.1 \%, 19.6 \%, 75.4 \%)$ \\
$(0.46,0.80)$ & $(45.3 \%, 25.5 \%, 75.7 \%)$ & $(58.9 \%,-5.0 \%, 74.5 \%)$ & $(47.0 \%, 21.9 \%, 75.6 \%)$ \\
$(0.47,0.72)$ & $(45.3 \%, 25.0 \%, 75.7 \%)$ & $(51.5 \%, 6.3 \%, 75.2 \%)$ & $(46.1 \%, 22.7 \%, 75.7 \%)$ \\
$(0.48,0.65)$ & $(45.3 \%, 24.4 \%, 75.7 \%)$ & $(47.9 \%, 13.6 \%, 75.5 \%)$ & $(45.6 \%, 23.1 \%, 75.7 \%)$ \\
$(0.49,0.57)$ & $(45.4 \%, 23.9 \%, 75.7 \%)$ & $(46.1 \%, 18.9 \%, 75.7 \%)$ & $(45.4 \%, 23.3 \%, 75.7 \%)$ \\
$(\mathbf{0 . 5 0}, \mathbf{0 . 5 0})$ & $(\mathbf{4 5 . 4 \% , 2 3 . 3 \% , 7 5 . 7 \% )}$ & $(\mathbf{4 5 . 4 \%}, \mathbf{2 3 . 3 \%}, \mathbf{7 5 . 7 \%})$ & $(\mathbf{4 5 . 4 \%}, \mathbf{2 3 . 3 \% , 7 5 . 7 \% )}$ \\
$(0.51,0.43)$ & $(45.4 \%, 22.8 \%, 75.7 \%)$ & $(45.4 \%, 27.3 \%, 75.7 \%)$ & $(45.4 \%, 23.3 \%, 75.7 \%)$ \\
$(0.52,0.35)$ & $(45.5 \%, 22.2 \%, 75.7 \%)$ & $(46.1 \%, 30.9 \%, 75.7 \%)$ & $(45.6 \%, 23.2 \%, 75.7 \%)$ \\
$(0.53,0.28)$ & $(45.6 \%, 21.6 \%, 75.7 \%)$ & $(47.1 \%, 34.6 \%, 75.6 \%)$ & $(45.8 \%, 23.2 \%, 75.7 \%)$ \\
$(0.54,0.20)$ & $(45.7 \%, 21.0 \%, 75.7 \%)$ & $(48.7 \%, 38.3 \%, 75.4 \%)$ & $(46.0 \%, 23.1 \%, 75.7 \%)$ \\
$(0.55,0.13)$ & $(45.8 \%, 20.4 \%, 75.7 \%)$ & $(50.7 \%, 42.2 \%, 75.2 \%)$ & $(46.3 \%, 23.0 \%, 75.6 \%)$ \\
$(0.56,0.06)$ & $(45.9 \%, 19.8 \%, 75.7 \%)$ & $(53.2 \%, 46.5 \%, 75.0 \%)$ & $(46.8 \%, 23.0 \%, 75.6 \%)$ \\
\hline \hline
\end{tabular}

Panel A: portfolio proportions $\left(\left(\pi_{t}^{M}\right)^{*} / x,\left(\pi_{t}^{A}\right)^{*} / x,\left(\pi_{t}^{B}\right)^{*} / x\right)$ according to correlation pairs $\left(\tilde{\rho}_{0}^{A}, \tilde{\rho}_{1}^{A}\right)$

\begin{tabular}{cccc}
\hline \hline Correlation Pairs & Portfolio Proportions (Regime 0) & Portfolio Proportions (Regime 1) & Weighted Average Portfolio Proportions \\
\hline$(0.04,0.54)$ & $(51.7 \%, 19.6 \%, 78.1 \%)$ & $(-9.1 \%, 55.4 \%, 84.7 \%)$ & $(44.5 \%, 23.9 \%, 78.8 \%)$ \\
$(0.05,0.47)$ & $(50.6 \%, 20.3 \%, 77.6 \%)$ & $(4.0 \%, 47.6 \%, 78.2 \%)$ & $(45.1 \%, 23.5 \%, 77.7 \%)$ \\
$(0.06,0.40)$ & $(49.6 \%, 20.9 \%, 77.2 \%)$ & $(14.2 \%, 41.7 \%, 74.7 \%)$ & $(45.3 \%, 23.4 \%, 76.9 \%)$ \\
$(0.07,0.32)$ & $(48.5 \%, 21.5 \%, 76.8 \%)$ & $(22.8 \%, 36.6 \%, 73.1 \%)$ & $(45.4 \%, 23.3 \%, 76.4 \%)$ \\
$(0.08,0.25)$ & $(47.5 \%, 22.1 \%, 76.4 \%)$ & $(30.5 \%, 32.1 \%, 72.9 \%)$ & $(45.4 \%, 23.3 \%, 76.0 \%)$ \\
$(0.09,0.17)$ & $(46.4 \%, 22.7 \%, 76.1 \%)$ & $(37.9 \%, 27.7 \%, 73.8 \%)$ & $(45.4 \%, 23.3 \%, 75.8 \%)$ \\
$(\mathbf{0 . 1 0}, \mathbf{0 . 1 0})$ & $(\mathbf{4 5 . 4 \% , 2 3 . 3 \% , 7 5 . 7 \% )}$ & $(\mathbf{4 5 . 4 \% , 2 3 . 3 \% , 7 5 . 7 \% )}$ & $(\mathbf{4 5 . 4 \% , 2 3 . 3 \% , 7 5 . 7 \% )}$ \\
$(0.11,0.03)$ & $(44.4 \%, 23.9 \%, 75.4 \%)$ & $(53.2 \%, 18.7 \%, 78.7 \%)$ & $(45.4 \%, 23.3 \%, 75.8 \%)$ \\
$(0.12,-0.05)$ & $(43.3 \%, 24.5 \%, 75.1 \%)$ & $(61.7 \%, 13.7 \%, 82.8 \%)$ & $(45.5 \%, 23.2 \%, 76.0 \%)$ \\
$(0.13,-0.12)$ & $(42.3 \%, 25.1 \%, 74.8 \%)$ & $(71.4 \%, 8.1 \%, 88.2 \%)$ & $(45.8 \%, 23.1 \%, 76.4 \%)$ \\
$(0.14,-0.20)$ & $(41.3 \%, 25.7 \%, 74.6 \%)$ & $(82.5 \%, 1.5 \%, 95.2 \%)$ & $(46.2 \%, 22.8 \%, 77.0 \%)$ \\
$(0.15,-0.27)$ & $(40.3 \%, 26.3 \%, 74.3 \%)$ & $(96.0 \%,-6.4 \%, 104.3 \%)$ & $(47.0 \%, 22.4 \%, 77.9 \%)$ \\
$(0.16,-0.34)$ & $(39.3 \%, 26.9 \%, 74.1 \%)$ & $(112.9 \%,-16.3 \%, 116.4 \%)$ & $(48.1 \%, 21.8 \%, 79.1 \%)$ \\
\hline \hline
\end{tabular}

Panel B: portfolio proportions $\left(\left(\pi_{t}^{M}\right)^{*} / x,\left(\pi_{t}^{A}\right)^{*} / x,\left(\pi_{t}^{B}\right)^{*} / x\right)$ according to correlation pairs $\left(\tilde{\rho}_{0}^{B}, \tilde{\rho}_{1}^{B}\right)$

Note. In the table, the weighted average of portfolio proportions in regime 0 and regime 1 by the average fraction of time spent in regime 0 and regime 1 yield the values of weighted average portfolio proportions. The portfolio proportions given by bold type represent the portfolio proportions for the baseline parameter values. The baseline parameter values are as follows: risk-free interest rate $r_{0}=r_{1}=0.01$, expected rate of stock returns $\mu_{0}^{M}=$ $\mu_{1}^{M}=0.08, \mu_{0}^{A}=\mu_{1}^{B}=0.05$, and $\mu_{0}^{B}=\mu_{1}^{B}=0.11$, stock volatilities $\sigma_{0}^{M}=\sigma_{1}^{M}=0.235$, $\sigma_{0}^{A}=\sigma_{1}^{A}=0.2$, and $\sigma_{0}^{B}=\sigma_{1}^{B}=0.25$, relative risk aversion $\gamma=2$, regime intensities $\lambda_{0}=0.2353$ and $\lambda_{1}=1.7391$, correlation between industry stocks $\rho_{i}=0$, and correlations between market portfolio and industry stocks $\tilde{\rho}_{i}^{A}=0.5$ and $\tilde{\rho}_{i}^{B}=0.1$ for regime $i \in\{0,1\}$. 
Table C.7: Table: Portfolio proportions according to correlation pairs.

\begin{tabular}{cccc}
\hline \hline Correlation Pairs & Portfolio Proportions (Regime 0) & Portfolio Proportions (Regime 1) & Weighted Average Portfolio Proportions \\
\hline$(0.21,0.97)$ & $(30.3 \%, 74.9 \%)$ & $(-680.0 \%, 605.1 \%)$ & $(-54.3 \%, 138.1 \%)$ \\
$(0.23,0.82)$ & $(28.5 \%, 74.8 \%)$ & $(-95.6 \%, 142.5 \%)$ & $(13.7 \%, 82.8 \%)$ \\
$(0.25,0.67)$ & $(26.7 \%, 74.7 \%)$ & $(-30.7 \%, 96.5 \%)$ & $(19.8 \%, 77.3 \%)$ \\
$(0.27,0.52)$ & $(24.8 \%, 74.6 \%)$ & $(-3.0 \%, 81.2 \%)$ & $(21.5 \%, 75.4 \%)$ \\
$(0.29,0.37)$ & $(22.9 \%, 74.7 \%)$ & $(14.7 \%, 75.6 \%)$ & $(\mathbf{2 2 . 0 \%}, \mathbf{7 4 . 7 \% )}$ \\
$(\mathbf{0 . 3 0}, \mathbf{0 . 3 0})$ & $(\mathbf{2 2 . 0 \%}, \mathbf{7 4 . 7 \%})$ & $(\mathbf{2 2 . 0 \%}, \mathbf{7 4 . 7 \% )}$ & $(22.0 \%, 74.8 \%)$ \\
$(0.31,0.23)$ & $(21.0 \%, 74.8 \%)$ & $(28.9 \%, 74.8 \%)$ & $(21.9 \%, 75.2 \%)$ \\
$(0.33,0.08)$ & $(19.1 \%, 75.0 \%)$ & $(42.4 \%, 77.3 \%)$ & $(21.9 \%, 76.2 \%)$ \\
$(0.35,-0.07)$ & $(17.1 \%, 75.2 \%)$ & $(57.2 \%, 83.2 \%)$ & $(22.2 \%, 77.6 \%)$ \\
$(0.37,-0.22)$ & $(15.1 \%, 75.5 \%)$ & $(75.3 \%, 93.1 \%)$ & $(23.3 \%, 79.9 \%)$ \\
$(0.39,-0.37)$ & $(13.0 \%, 76.0 \%)$ & $(99.8 \%, 109.2 \%)$ & \\
\hline \hline
\end{tabular}

Panel A: portfolio proportions $\left(\left(\pi_{t}^{A}\right)^{*} / x,\left(\pi_{t}^{B}\right)^{*} / x\right)$ according to correlation pairs $\left(\rho_{0}, \rho_{1}\right)$ when $\tilde{\rho}_{i}^{k}=0$ for Industry $k \in\{A, B\}$ in regime $i \in\{0,1\}$

\begin{tabular}{cccc}
\hline \hline Correlation Pairs & Portfolio Proportions (Regime 0) & Portfolio Proportions (Regime 1) & Weighted Average Portfolio Proportions \\
\hline$(0.21,0.97)$ & $(56.7 \%,-3.0 \%, 102.0 \%)$ & $(-207.5 \%, 758.0 \%,-687.8 \%)$ & $(251.8 \%, 87.7 \%, 7.8 \%)$ \\
$(0.23,0.82)$ & $(57.7 \%,-5.7 \%, 102.7 \%)$ & $(192.1 \%,-380.0 \%, 435.4 \%)$ & $(73.7 \%,-50.3 \%, 142.3 \%)$ \\
$(0.25,0.67)$ & $(58.8 \%,-8.4 \%, 103.5 \%)$ & $(101.8 \%,-124.6 \%, 190.7 \%)$ & $(64.0 \%,-22.2 \%, 113.9 \%)$ \\
$(0.27,0.52)$ & $(59.9 \%,-11.1 \%, 104.4 \%)$ & $(78.4 \%,-59.7 \%, 134.5 \%)$ & $(62.1 \%,-16.9 \%, 108.0 \%)$ \\
$(0.29,0.37)$ & $(61.1 \%,-14.0 \%, 105.5 \%)$ & $(66.2 \%,-27.2 \%, 112.0 \%)$ & $(61.7 \%,-15.6 \%, 106.3 \%)$ \\
$(\mathbf{0 . 3 0}, \mathbf{0 . 3 0})$ & $(\mathbf{6 1 . 7 \%}, \mathbf{- 1 5 . 4 \%}, \mathbf{1 0 6 . 1 \% )}$ & $(\mathbf{6 1 . 7 \%}, \mathbf{- 1 5 . 4 \% , 1 0 6 . 1 \% )}$ & $(\mathbf{6 1 . 7 \%}, \mathbf{- 1 5 . 4 \% , 1 0 6 . 1 \% )}$ \\
$(0.31,0.23)$ & $(62.2 \%,-16.9 \%, 106.8 \%)$ & $(57.5 \%,-5.1 \%, 102.5 \%)$ & $(61.7 \%,-15.5 \%, 106.3 \%)$ \\
$(0.33,0.08)$ & $(63.4 \%,-20.0 \%, 108.2 \%)$ & $(49.7 \%, 13.5 \%, 100.4 \%)$ & $(61.8 \%,-16.0 \%, 107.3 \%)$ \\
$(0.35,-0.07)$ & $(64.7 \%,-23.2 \%, 109.8 \%)$ & $(41.2 \%, 32.5 \%, 104.3 \%)$ & $(61.9 \%,-16.5 \%, 109.2 \%)$ \\
$(0.37,-0.22)$ & $(66.0 \%,-26.5 \%, 111.6 \%)$ & $(30.4 \%, 55.7 \%, 115.4 \%)$ & $(61.7 \%,-16.7 \%, 112.1 \%)$ \\
$(0.39,-0.37)$ & $(67.3 \%,-30.0 \%, 113.7 \%)$ & $(14.1 \%, 89.6 \%, 138.5 \%)$ & $(61.0 \%,-15.8 \%, 116.69 \%)$ \\
\hline \hline
\end{tabular}

Panel B: portfolio proportions $\left(\left(\pi_{t}^{M}\right)^{*} / x,\left(\pi_{t}^{A}\right)^{*} / x,\left(\pi_{t}^{B}\right)^{*} / x\right)$ according to correlation pairs $\left(\rho_{0}, \rho_{1}\right)$ when $\tilde{\rho}_{i}^{A}=0.5$ and $\tilde{\rho}_{i}^{B}=0.1$ in regime $i \in\{0,1\}$

Note. In the table, the weighted average of portfolio proportions in regime 0 and regime 1 by the average fraction of time spent in regime 0 and regime 1 yield the values of weighted average portfolio proportions. In Panel A, we do not report stock holdings in market portfolio because they are invariant with correlation pairs. The portfolio proportions given by bold type represent the portfolio proportions for the baseline parameter values. The baseline parameter values are as follows: risk-free interest rate $r_{0}=r_{1}=0.01$, expected rate of stock returns $\mu_{0}^{M}=\mu_{1}^{M}=0.08, \mu_{0}^{A}=\mu_{1}^{B}=0.05$, and $\mu_{0}^{B}=\mu_{1}^{B}=0.11$, stock volatilities $\sigma_{0}^{M}=\sigma_{1}^{M}=0.235, \sigma_{0}^{A}=\sigma_{1}^{A}=0.2$, and $\sigma_{0}^{B}=\sigma_{1}^{B}=0.25$, relative risk aversion $\gamma=2$, regime intensities $\lambda_{0}=0.2353$ and $\lambda_{1}=1.7391$, and correlation between industry stocks $\rho_{i}=0.3$ for regime $i \in\{0,1\}$. 